Article

\title{
Construction of Stancu-Type Bernstein Operators Based on Bézier Bases with Shape Parameter $\lambda$
}

\author{
Hari M. Srivastava ${ }^{1,2, *} \mathbb{( \mathbb { D }}$, Faruk Özger $^{3}{ }^{\mathbb{D}}$ and S. A. Mohiuddine ${ }^{4}$ \\ 1 Department of Mathematics and Statistics, University of Victoria, Victoria, BC V8W 3R4, Canada \\ 2 Department of Medical Research, China Medical University Hospital, China Medical University, \\ Taichung 40402, Taiwan \\ 3 Department of Engineering Sciences, İzmir Katip Çelebi University, İzmir 35620, Turkey; \\ farukozger@gmail.com \\ 4 Operator Theory and Applications Research Group, Department of Mathematics, Faculty of Science, \\ King Abdulaziz University, P.O. Box 80203, Jeddah 21589, Saudi Arabia; mohiuddine@gmail.com \\ * Correspondence: harimsri@math.uvic.ca
}

Received: 10 February 2019; Accepted: 25 February 2019; Published: 2 March 2019

\begin{abstract}
We construct Stancu-type Bernstein operators based on Bézier bases with shape parameter $\lambda \in[-1,1]$ and calculate their moments. The uniform convergence of the operator and global approximation result by means of Ditzian-Totik modulus of smoothness are established. Also, we establish the direct approximation theorem with the help of second order modulus of smoothness, calculate the rate of convergence via Lipschitz-type function, and discuss the Voronovskaja-type approximation theorems. Finally, in the last section, we construct the bivariate case of Stancu-type $\lambda$-Bernstein operators and study their approximation behaviors.
\end{abstract}

Keywords: Stancu-type Bernstein operators; Bézier bases; Voronovskaja-type theorems; modulus of continuity; rate of convergence; bivariate operators; approximation properties

MSC: 41A25; 41A35

\section{Introduction}

A famous mathematician Bernstein [1] constructed polynomials nowadays called Bernstein polynomials, which are familiar and widely investigated polynomials in theory of approximation. Bernstein gave a simple and very elegant way to obtain Weierstrass approximation theorem with the help of his newly constructed polynomials. For any continuous function $f(x)$ defined on $C[0,1]$, Bernstein polynomials of order $n$ are given by

$$
B_{n}(f ; x)=\sum_{i=0}^{n} f\left(\frac{i}{n}\right) b_{n, i}(x) \quad(x \in[0,1]),
$$

where the Bernstein basis functions $b_{n, i}(x)$ are defined by

$$
b_{n, i}(x)=\left(\begin{array}{c}
n \\
i
\end{array}\right) x^{i}(1-x)^{n-i} \quad(i=0, \ldots, n) .
$$

Stancu [2] presented a generalization of Bernstein polynomials with the help of two parameters $\alpha$ and $\beta$ such that $0 \leq \alpha \leq \beta$, as follows:

$$
S_{n, \alpha, \beta}(f ; x)=\sum_{i=0}^{n} f\left(\frac{i+\alpha}{n+\beta}\right)\left(\begin{array}{c}
n \\
i
\end{array}\right) x^{i}(1-x)^{n-i} \quad(x \in[0,1]) .
$$


If we take both the parameters $\alpha=\beta=0$, then we get the classical Bernstein polynomials. The operators defined by (2) are called Bernstein-Stancu operators. For some recent work, we refer to [3-6].

In the recent past, Cai et al. [7] presented a new construction of Bernstein operators with the help of Bézier bases with shape parameter $\lambda$ and called it $\lambda$-Bernstein operators, which are defined by

$$
B_{n}^{\lambda}(f ; x)=\sum_{i=0}^{n} f\left(\frac{i}{n}\right) \tilde{b}_{n, i}(\lambda ; x) \quad(n \in \mathbb{N})
$$

where $\tilde{b}_{n, i}(\lambda ; x)$ are Bézier bases with shape parameter $\lambda$ (see [8]), defined by

$$
\begin{aligned}
& \tilde{b}_{n, 0}(\lambda ; x)=b_{n, 0}(x)-\frac{\lambda}{n+1} b_{n+1,1}(x) \\
& \tilde{b}_{n, i}(\lambda ; x)=b_{n, i}(x)+\frac{n-2 i+1}{n^{2}-1} \lambda b_{n+1, i}(x)-\frac{n-2 i-1}{n^{2}-1} \lambda b_{n+1, i+1}(x), \quad i=1,2 \ldots, n-1, \\
& \tilde{b}_{n, n}(\lambda ; x)=b_{n, n}(x)-\frac{\lambda}{n+1} b_{n+1, n}(x)
\end{aligned}
$$

in this case $\lambda \in[-1,1]$ and $b_{n, i}(x)$ are the Bernstein basis functions. By taking the above operators into account, they established various approximation results, namely, Korovkin- and Voronovskaja-type theorems, rate of convergence via Lipschitz continuous functions, local approximation and other related results. In the same year, Cai [9] generalized $\lambda$-Bernstein operators by constructing the Kantorovich-type $\lambda$-Bernstein operators, as well as its Bézier variant, and studied several approximation results. Later, various approximation properties and asymptotic type results of the Kantorovich-type $\lambda$-Bernstein operators have been studied by Acu et al. [10]. Very recently, Özger [11] obtained statistical approximation for $\lambda$-Bernstein operators including a Voronovskaja-type theorem in statistical sense. In the same article, he also constructed bivariate $\lambda$-Bernstein operators and studied their approximation properties.

The Bernstein operators are some of the most studied positive linear operators which were modified by many authors, and we are mentioning some of them and other related work [12-23].

We are now ready to construct our new operators as follows: Suppose that $\alpha$ and $\beta$ are two non-negative parameters such that $0 \leq \alpha \leq \beta$. Then, the Stancu-type modification of $\lambda$-Bernstein operators $B_{n, \alpha, \beta}^{\lambda}(f ; x): C[0,1] \longrightarrow C[0,1]$ is defined by

$$
B_{n, \alpha, \beta}^{\lambda}(f ; x)=\sum_{i=0}^{n} f\left(\frac{i+\alpha}{n+\beta}\right) \tilde{b}_{n, i}(\lambda ; x)
$$

for any $n \in \mathbb{N}$ and we call it Stancu-type $\lambda$-Bernstein operators or $\lambda$-Bernstein-Stancu operators, where Bézier bases $\tilde{b}_{n, i}(\lambda ; x)$ are defined in (4).

Remark 1. We have the following results for Stancu-type $\lambda$-Bernstein operators:

(i) If we take $\lambda=0$ in (5), then Stancu-type $\lambda$-Bernstein Stancu operators reduce to the classical Bernstein-Stancu operators defined in [2].

(ii) The choice of $\alpha=\beta=0$ in (5) gives $\lambda$-Bernstein operators defined by Cai et al. [7].

(iii) If we choose $\alpha=\beta=\lambda=0$, then (5) reduces to the classical Bernstein operators defined in [1].

The rest of the paper is organized as follows: In Section 2, we calculate the moments of (5) and prove global approximation formula in terms of Ditzian-Totik uniform modulus of smoothness of first and second order. The local direct estimate of the rate of convergence by Lipschitz-type function involving two parameters for $\lambda$-Bernstein-Stancu operators is investigated. In Section 3 , we establish quantitative Voronovskaja-type theorem for our operators. The final section of the paper is devoted to study the bivariate case of $\lambda$-Bernstein-Stancu operators . 


\section{Some Auxiliary Lemmas and Approximation by Stancu-Type $\lambda$-Bernstein Operators}

In this section, we first prove some lemma which will be used to study the approximation results of (5).

Lemma 1. For $x \in[0,1]$, the moments of Stancu-type $\lambda$-Bernstein operators are given as:

$$
\begin{aligned}
B_{n, \alpha, \beta}^{\lambda}(1 ; x)= & 1 ; \\
B_{n, \alpha, \beta}^{\lambda}(t ; x)= & \frac{\alpha+n x}{n+\beta}+\lambda\left[\frac{1-2 x+x^{n+1}+(\alpha-1)(1-x)^{n+1}}{(n+\beta)(n-1)}+\frac{\alpha x(1-x)^{n}}{n+\beta}\right] \\
B_{n, \alpha, \beta}^{\lambda}\left(t^{2} ; x\right)= & \frac{1}{(n+\beta)^{2}}\left\{n(n-1) x^{2}+(1+2 \alpha) n x+\alpha^{2}\right\} \\
& +\lambda\left[\frac{2 n x-1-4 n x^{2}+(2 n+1) x^{n+1}+(1-x)^{n+1}}{(n+\beta)^{2}(n-1)}+\frac{\alpha^{2}-4 \alpha x}{(n+\beta)^{2}(n-1)}\right. \\
& \left.+\frac{2 \alpha n-2 \alpha(\alpha+n)\left(x^{n+1}+(1-x)^{n}\right)+\alpha^{2} x\left(n^{2}+1\right)(1-x)^{n}}{(n+\beta)^{2}\left(n^{2}-1\right)}\right] .
\end{aligned}
$$

Proof. Using the definition of operators (5) and Bézier-Bernstein bases $\tilde{b}_{n, i}(\lambda ; x)(4)$, we write

$$
\begin{aligned}
B_{n, \alpha, \beta}^{\lambda}(t ; x)= & \sum_{i=0}^{n} \frac{i+\alpha}{n+\beta} \tilde{b}_{n, i}(\lambda ; x)=\frac{\alpha}{n+\beta} b_{n, 0}(x)-\frac{\alpha}{n+\beta} \frac{\lambda}{n+1} b_{n+1,1}(x) \\
& +\sum_{i=1}^{n-1} \frac{i+\alpha}{n+\beta}\left[b_{n, i}(x)+\lambda\left(\frac{n-2 i+n+1}{n^{2}-1} b_{n+1, i}(x)-\frac{n-2 i-1}{n^{2}-1} b_{n+1, i+1}(x)\right)\right] \\
& +\frac{n+\alpha}{n+\beta} b_{n, n}(x)-\frac{n+\alpha}{n+\beta} \frac{\lambda}{n+1} b_{n+1, n}(x) \\
= & \sum_{i=0}^{n} \frac{i+\alpha}{n+\beta} b_{n, i}(x)+\lambda\left(\theta_{1}(n, \alpha, \beta, x)-\theta_{2}(n, \alpha, \beta, x)\right),
\end{aligned}
$$

where

$$
\begin{aligned}
& \theta_{1}(n, \alpha, \beta, x)=\sum_{i=0}^{n} \frac{i+\alpha}{n+\beta} \frac{n-2 i+1}{n^{2}-1} b_{n+1, i}(x) \\
& \theta_{2}(n, \alpha, \beta, x)=\sum_{i=1}^{n-1} \frac{i+\alpha}{n+\beta} \frac{n-2 i-1}{n^{2}-1} b_{n+1, i+1}(x) .
\end{aligned}
$$

Now, we compute the expressions $\theta_{1}(n, \alpha, \beta, x)$ and $\theta_{2}(n, \alpha, \beta, x)$. Since the Bernstein-Stancu operators are linear, and Bernstein-Stancu operators and fundamental Bernstein bases satisfy the following equality:

$$
\sum_{i=1}^{n} \frac{i+\alpha}{n+\beta} b_{n, i}(x)=\frac{n x}{n+\beta}+\frac{\alpha}{n+\beta}
$$

one writes

$$
\begin{aligned}
\theta_{1}(n, \alpha, \beta, x)= & \frac{1}{n-1} \sum_{i=0}^{n} \frac{i+\alpha}{n+\beta} b_{n+1, i}(x)-\frac{2}{n^{2}-1} \sum_{i=0}^{n} \frac{i^{2}+\alpha i}{n+\beta} b_{n+1, i}(x) \\
= & \frac{1}{n-1} \sum_{i=0}^{n} \frac{i}{n+\beta} b_{n+1, i}(x)+\frac{1}{n-1} \sum_{i=0}^{n} \frac{\alpha}{n+\beta} b_{n+1, i}(x) \\
& -\frac{2}{n^{2}-1} \sum_{i=1}^{n} \frac{i^{2}}{n+\beta} b_{n+1, i}(x)-\frac{2}{n^{2}-1} \sum_{i=1}^{n} \frac{\alpha i}{n+\beta} b_{n+1, i}(x)
\end{aligned}
$$




$$
\begin{aligned}
& =\frac{(n+1) x-2 x}{(n+\beta)(n-1)} \sum_{i=0}^{n-1} b_{n, i}(x)+\frac{\alpha}{(n+\beta)(n-1)} \sum_{i=0}^{n} b_{n+1, i}(x) \\
& -\frac{2 \alpha x}{(n+\beta)(n-1)} \sum_{i=0}^{n-1} b_{n, i}(x)-\frac{2 n x^{2}}{(n+\beta)(n-1)} \sum_{i=0}^{n-2} b_{n-1, i}(x) \\
& =\frac{x-x^{n+d+1}}{n+\beta}-\frac{2 n x^{2}-2 n x^{n+1}}{(n+\beta)(n-1)}+\frac{x-x^{n+d+1}}{n+\beta}-\frac{\alpha-2 \alpha x+\alpha x^{n+1}}{(n+\beta)(n-1)} \\
& \theta_{2}(n, \alpha, \beta, x)=\frac{1}{n+1} \sum_{i=1}^{n-1} \frac{i+\alpha}{n+\beta} b_{n+1, i+1}(x)-\frac{2}{n^{2}-1} \sum_{i=1}^{n-1} \frac{i^{2}+\alpha i}{n+\beta} b_{n+1, i+1}(x) \\
& =\frac{1}{n+1} \sum_{i=1}^{n-1} \frac{i}{n+\beta} b_{n+1, i+1}(x)+\frac{1}{n+1} \sum_{i=1}^{n-1} \frac{\alpha}{n+\beta} b_{n+1, i+1}(x) \\
& -\frac{2}{n^{2}-1} \sum_{i=1}^{n-1} \frac{i^{2}}{n+\beta} b_{n+1, i+1}(x)-\frac{2}{n^{2}-1} \sum_{i=1}^{n-1} \frac{\alpha i}{n+\beta} b_{n+1, i+1}(x) \\
& =\frac{x}{n+\beta} \sum_{i=1}^{n-1} b_{n, i}(x)-\frac{1}{(n+\beta)(n+1)} \sum_{i=1}^{n-1} b_{n+1, i+1}(x) \\
& -\frac{2 n x^{2}}{(n+\beta)(n+1)} \sum_{i=0}^{n-2} b_{n-1, i}(x)+\frac{2 x}{(n+\beta)(n+1)} \sum_{i=1}^{n-1} b_{n, i}(x) \\
& -\frac{2}{(n+\beta)\left(n^{2}-1\right)} \sum_{i=1}^{n-1} b_{n+1, i+1}(x)+\frac{\alpha}{(n+\beta)(n+1)} \sum_{i=1}^{n-1} b_{n+1, i+1}(x) \\
& -\frac{2 \alpha x}{(n+\beta)(n-1)} \sum_{i=1}^{n-1} b_{n, i}(x)+\frac{2 \alpha}{(n+\beta)\left(n^{2}-1\right)} \sum_{i=1}^{n-1} b_{n+1, i+1}(x) \\
& =\frac{x-x^{n+1}}{n+\beta}-\frac{x(1-x)^{n}}{n+\beta}-\frac{1-(1-x)^{n+1}-x(n+1)(1-x)^{n}-x^{n+1}}{(n+\beta)(n+1)} \\
& -\frac{2-(1-x)^{n+1}-2 x(n+1)(1-x)^{n}-2 x^{n+1}}{(n+\beta)\left(n^{2}-1\right)}+\frac{\alpha-\alpha(1-x)^{n+1}-\alpha x^{n+1}}{(n+\beta)(n+1)} \\
& +\frac{2 x-2 x(1-x)^{n}-2 x^{n+1}}{(n+\beta)(n-1)}-\frac{2 n x^{2}-2 n x^{n+1}}{(n+\beta)(n-1)}-\frac{\alpha x(1-x)^{n}}{n+\beta} \\
& -\frac{2 \alpha x-2 \alpha x^{n+1}}{(n+\beta)(n+1)}+\frac{2 \alpha-2 \alpha(1-x)^{n+1}-2 \alpha x^{n+1}}{(n+\beta)\left(n^{2}-1\right)} \text {. }
\end{aligned}
$$

We get the desired result for $B_{n, \alpha, \beta}^{\lambda}(t ; x)$ by combining the results obtained for $\theta_{1}(n, \alpha, \beta, x)$ and $\theta_{2}(n, \alpha, \beta, x)$.

Again, by using the following identity;

$$
\sum_{i=1}^{n} \frac{(i+\alpha)^{2}}{(n+\beta)^{2}} b_{n, i}(x)=\frac{1}{(n+\beta)^{2}}\left\{n(n-1) x^{2}+(1+2 \alpha) n x+\alpha^{2}\right\}
$$

together with (4) and (5), we can write

$$
\begin{aligned}
B_{n, \alpha, \beta}^{\lambda}\left(t^{2} ; x\right)= & \sum_{i=0}^{n} \frac{(i+\alpha)^{2}}{(n+\beta)^{2}} \tilde{b}_{n, i}(\lambda ; x)=\frac{\alpha^{2}}{(n+\beta)^{2}} b_{n, 0}(x)-\frac{\alpha^{2}}{(n+\beta)^{2}} \frac{\lambda}{n+1} b_{n+1,1}(x) \\
& +\sum_{i=1}^{n-1} \frac{(i+\alpha)^{2}}{(n+\beta)^{2}}\left[b_{n, i}(x)+\lambda\left(\frac{n-2 i+1}{n^{2}-1} b_{n+1, i}(x)-\frac{n-2 i-1}{n^{2}-1} b_{n+1, i+1}(x)\right)\right] \\
& +\frac{(n+\alpha)^{2}}{(n+\beta)^{2}} b_{n, n}(x)-\frac{(n+\alpha)^{2}}{(n+\beta)^{2}} \frac{\lambda}{n+1} b_{n+1, n}(x)
\end{aligned}
$$




$$
=\sum_{i=0}^{n} \frac{(i+\alpha)^{2}}{(n+\beta)^{2}} b_{n, i}(x)+\lambda\left(\theta_{3}(n, \alpha, \beta, x)-\theta_{4}(n, \alpha, \beta, x)\right),
$$

where

$$
\begin{aligned}
& \theta_{3}(n, \alpha, \beta, x)=\sum_{i=0}^{n} \frac{(i+\alpha)^{2}}{(n+\beta)^{2}} \frac{n-2 i+1}{n^{2}-1} b_{n+1, i}(x) \\
& \theta_{4}(n, \alpha, \beta, x)=\sum_{i=1}^{n-1} \frac{(i+\alpha)^{2}}{(n+\beta)^{2}} \frac{n-2 i-1}{n^{2}-1} b_{n+1, i+1}(x) .
\end{aligned}
$$

We now compute the expressions $\theta_{3}(n, \alpha, \beta, x)$ and $\theta_{4}(n, \alpha, \beta, x)$ as follows:

$$
\begin{aligned}
& \theta_{3}(n, \alpha, \beta, x)=\frac{1}{n-1} \sum_{i=0}^{n} \frac{(i+\alpha)^{2}}{(n+\beta)^{2}} b_{n+1, i}(x)-\frac{2}{n^{2}-1} \sum_{i=0}^{n} \frac{(i+\alpha)^{2} i}{(n+\beta)^{2}} b_{n+1, i}(x) \\
& =\frac{1}{n-1} \sum_{i=0}^{n} \frac{i^{2}}{(n+\beta)^{2}} b_{n+1, i}(x)+\frac{2 \alpha}{n-1} \sum_{i=0}^{n} \frac{i}{(n+\beta)^{2}} b_{n+1, i}(x) \\
& +\frac{\alpha^{2}}{n-1} \sum_{i=0}^{n} b_{n+1, i}(x)-\frac{2}{n^{2}-1} \sum_{i=0}^{n} \frac{i^{3}}{(n+\beta)^{2}} b_{n+1, i}(x) \\
& -\frac{4 \alpha}{n^{2}-1} \sum_{i=0}^{n} \frac{i^{2}}{(n+\beta)^{2}} b_{n+1, i}(x)-\frac{2 \alpha^{2}}{n^{2}-1} \sum_{i=0}^{n} \frac{i}{(n+\beta)^{2}} b_{n+1, i}(x) \\
& =\frac{n(n+1) x^{2}}{(n+\beta)^{2}(n-1)} \sum_{i=0}^{n-2} b_{n-1, i}(x)+\frac{(n+1) x}{(n+\beta)^{2}(n-1)} \sum_{i=0}^{n-1} b_{n, i}(x) \\
& -\frac{2 n x^{3}}{(n+\beta)^{2}} \sum_{i=0}^{n-3} b_{n-2, i}(x)-\frac{6 n x^{2}}{(n+\beta)^{2}(n-1)} \sum_{i=0}^{n-2} b_{n-1, i}(x) \\
& -\frac{x}{(n+\beta)^{2}(n-1)} \sum_{i=0}^{n-1} b_{n, i}(x)+\frac{2 \alpha x(n+1)}{(n+\beta)^{2}(n-1)} \sum_{i=0}^{n-1} b_{n, i}(x) \\
& +\frac{\alpha^{2}}{(n+\beta)^{2}(n-1)} \sum_{i=0}^{n} b_{n+1, i}(x)-\frac{4 \alpha n x^{2}}{(n+\beta)^{2}(n-1)} \sum_{i=0}^{n-2} b_{n-1, i}(x) \\
& -\frac{4 \alpha x}{(n+\beta)^{2}(n-1)} \sum_{i=0}^{n-1} b_{n, i}(x)-\frac{2 \alpha^{2} x}{(n+\beta)^{2}(n-1)} \sum_{i=0}^{n-1} b_{n, i}(x) \\
& =\frac{2 n\left(x^{n+1}-x^{3}\right)}{(n+\beta)^{2}}+\frac{x-x^{n+1}}{(n+\beta)^{2}}+\frac{\left(n^{2}-5 n\right)\left(x^{2}-x^{n+1}\right)}{(n+\beta)^{2}(n-1)} \\
& +\frac{2 \alpha(n+1) x^{n+1}+\alpha^{2}\left(1-x+x^{n+1}\right)-4 \alpha n x^{2}}{(n+\beta)^{2}(n-1)}+\frac{2 \alpha x}{(n+\beta)^{2}} \text {. } \\
& \theta_{4}(n, \alpha, \beta, x)=\frac{1}{n+1} \sum_{i=1}^{n-1} \frac{(i+\alpha)^{2}}{(n+\beta)^{2}} b_{n+1, i+1}(x)-\frac{2}{n^{2}-1} \sum_{i=1}^{n-1} \frac{(i+\alpha)^{2} i}{(n+\beta)^{2}} b_{n+1, i+1}(x) \\
& =\frac{1}{n+1} \sum_{i=1}^{n-1} \frac{i^{2}}{(n+\beta)^{2}} b_{n+1, i+1}(x)+\frac{2 \alpha}{n+1} \sum_{i=1}^{n-1} \frac{i}{(n+\beta)^{2}} b_{n+1, i+1}(x) \\
& +\frac{\alpha^{2}}{n+1} \sum_{i=1}^{n-1} \frac{1}{(n+\beta)^{2}} b_{n+1, i+1}(x)-\frac{2}{n^{2}-1} \sum_{i=1}^{n-1} \frac{i^{3}}{(n+\beta)^{2}} b_{n+1, i+1}(x) \\
& -\frac{4 \alpha}{n^{2}-1} \sum_{i=1}^{n-1} \frac{i^{2}}{(n+\beta)^{2}} b_{n+1, i+1}(x)-\frac{2 \alpha^{2}}{n^{2}-1} \sum_{i=1}^{n-1} \frac{i}{(n+\beta)^{2}} b_{n+1, i+1}(x)
\end{aligned}
$$




$$
\begin{aligned}
= & \frac{n x^{2}}{(n+\beta)^{2}} \sum_{i=0}^{n-2} b_{n-1, i}(x)+\frac{1}{(n+\beta)^{2}(n-1)} \sum_{i=1}^{n-1} b_{n+1, i+1}(x) \\
& -\frac{2 n x^{3}}{(n+\beta)^{2}} \sum_{i=0}^{n-3} b_{n-2, i}(x)-\frac{-2 x}{(n+\beta)^{2}(n-1)} \sum_{i=1}^{n-1} b_{n, i}(x) \\
& +\frac{2}{(n+\beta)^{2}(n-1)} \sum_{i=1}^{n-1} b_{n+1, i+1}(x)-\frac{x}{(n+\beta)^{2}} \sum_{i=1}^{n-1} b_{n, i}(x) \\
& +\frac{2 \alpha x}{(n+\beta)^{2}} \sum_{i=1}^{n-1} b_{n, i}(x)-\frac{2 \alpha}{(n+\beta)^{2}(n+1)} \sum_{i=1}^{n-1} b_{n+1, i+1}(x) \\
& +\frac{\alpha^{2}}{(n+\beta)^{2}(n+1)} \sum_{i=1}^{n-1} b_{n+1, i+1}(x)-\frac{4 \alpha n x^{2}}{(n+\beta)^{2}(n-1)} \sum_{i=0}^{n-2} b_{n-1, i}(x) \\
& +\frac{4 \alpha x}{(n+\beta)^{2}(n-1)} \sum_{i=1}^{n-1} b_{n, i}(x)-\frac{4 \alpha}{(n+\beta)^{2}\left(n^{2}-1\right)} \sum_{i=1}^{n-1} b_{n+1, i+1}(x) \\
& -\frac{2 \alpha^{2} x}{(n+\beta)^{2}(n-1)} \sum_{i=1}^{n-1} b_{n, i}(x)+\frac{2 \alpha}{(n+\beta)^{2}\left(n^{2}-1\right)} \sum_{i=1}^{n-1} b_{n+1, i+1}(x) \\
= & \frac{n x^{2}+(n+1) x^{n+1}-x-2 n x^{3}}{(n+\beta)^{2}}+\frac{1-(1-x)^{n+1}-x^{n+1}}{(n+\beta)^{2}(n+1)} \\
& +\frac{2 x^{n+1}-2 x}{(n+\beta)^{2}(n-1)}+\frac{2-2(1-x)^{n+1}-2 x^{n+1}}{(n+\beta)^{2}\left(n^{2}-1\right)} \\
& +\frac{2 \alpha x+2 \alpha x^{n+1}-\alpha^{2} x(1-x)^{n}}{(n+\beta)^{2}}+\frac{\alpha(\alpha-2)\left(1-x^{n+1}-(1-x)^{n+1}\right)}{(n+\beta)^{2}(n+1)} \\
& +\frac{2 \alpha(\alpha-2) x\left((1-x)^{n+1}-1\right)+2 \alpha^{2} x^{n+1}}{(n+\beta)^{2}(n-1)}+\frac{2 \alpha\left(x^{n+1}+(1-x)^{n+1}-1\right)}{(n+\beta)^{2}\left(n^{2}-1\right)}
\end{aligned}
$$

which completes the result for $B_{n, \alpha, \beta}^{\lambda}\left(t^{2} ; x\right)$ by combining the results obtained for $\theta_{3}(n, \alpha, \beta, x)$ and $\theta_{4}(n, \alpha, \beta, x)$.

Corollary 1. The following relations hold:

$$
\begin{aligned}
B_{n, \alpha, \beta}^{\lambda}(t-x ; x)= & \sum_{i=0}^{n} \frac{i+\alpha}{n+\beta} \tilde{b}_{n, i}(\lambda ; x)-x \sum_{i=0}^{n} \tilde{b}_{n, i}(\lambda ; x) \\
= & \frac{\alpha-\beta x}{n+\beta}+\lambda \frac{1-2 x+x^{n+1}-(1-x)^{n+1}}{(n+\beta)(n-1)} \\
& +\lambda \frac{\alpha x(1-x)^{n}}{n+\beta}+\lambda \frac{\alpha(1-x)^{n+1}}{(n+\beta)(n-1)} ; \\
B_{n, \alpha, \beta}^{\lambda}\left((t-x)^{2} ; x\right)= & \sum_{i=0}^{n}\left(\frac{i+\alpha}{n+\beta}\right)^{2} \tilde{b}_{n, i}(\lambda ; x)-2 x \sum_{i=0}^{n} \frac{i+\alpha}{n+\beta} \tilde{b}_{n, i}(\lambda ; x)+x^{2} \sum_{i=0}^{n} \tilde{b}_{n, i}(\lambda ; x) \\
= & \frac{n x(1-x)+(\beta x-\alpha)^{2}}{(n+\beta)^{2}} \\
& +\lambda\left[\frac{4 x^{2}-2 x-2 x^{n+2}-2(\alpha-1) x(1-x)^{n+1}}{(n+\beta)(n-1)}-\frac{2 \alpha x^{2}(1-x)^{n}}{n+\beta}\right] \\
& +\lambda \frac{2 n x-1-4 n x^{2}+(2 n+1) x^{n+1}+(1-x)^{n+1}+\alpha^{2}-4 \alpha x}{(n+\beta)^{2}(n-1)} \\
& +\lambda \frac{2 \alpha n-2 \alpha(\alpha+n)\left(x^{n+1}+(1-x)^{n}\right)+\alpha^{2} x\left(n^{2}+1\right)(1-x)^{n}}{(n+\beta)^{2}\left(n^{2}-1\right)} .
\end{aligned}
$$


Corollary 2. The following identities hold:

$$
\begin{aligned}
& \lim _{n \rightarrow \infty} n B_{n, \alpha, \beta}^{\lambda}(t-x ; x ;)=\alpha-\beta x ; \\
& \lim _{n \rightarrow \infty} n B_{n, \alpha, \beta}^{\lambda}\left((t-x)^{2} ; x\right)=x(1-x) .
\end{aligned}
$$

We obtain the uniform convergence of operators $B_{n, \alpha, \beta}^{\lambda}(f ; x)$ by applying well-known Bohman-Korovkin-Popoviciu theorem.

Theorem 1. Let $C[0,1]$ denote the space of all real-valued continuous functions on $[0,1]$ endowed with the supremum norm. Then

$$
\lim _{n \rightarrow \infty} B_{n, \alpha, \beta}^{\lambda}(f ; x)=f(x) \quad(f \in C[0,1])
$$

uniformly in $[0,1]$

Proof. It is sufficient to show that

$$
\lim _{n \rightarrow \infty}\left\|B_{n, \alpha, \beta}^{\lambda}\left(t^{j} ; x\right)-t^{j}\right\|_{C[0,1]}=0, \quad j=0,1,2
$$

as stated in Bohman-Korovkin-Popoviciu theorem. We have the following relations by Lemma 1:

$$
\lim _{n \rightarrow \infty}\left\|B_{n, \alpha, \beta}^{\lambda}\left(t^{0} ; x\right)-t^{0}\right\|_{C[0,1]}=0 \quad \text { and } \quad \lim _{n \rightarrow \infty}\left\|B_{n, \alpha, \beta}^{\lambda}(t ; x)-t\right\|_{C[0,1]}=0 .
$$

It is easy to show

$$
\begin{aligned}
B_{n, \alpha, \beta}^{\lambda}\left(t^{2} ; x\right) \leq & \frac{n(n+1) x^{2}+(1+2 \alpha) n x+\alpha^{2}}{(n+\beta)^{2}} \\
& +\lambda\left[\frac{2 n x+1+4 n x^{2}+(2 n+1) x^{n+1}+(1-x)^{n+1}}{(n+\beta)^{2}(n-1)}+\frac{\alpha^{2}+4 \alpha x}{(n+\beta)^{2}(n-1)}\right. \\
& \left.+\frac{2 \alpha n+2 \alpha(\alpha+n)\left(x^{n+1}+(1-x)^{n}\right)+\alpha^{2}\left(n^{2}+1\right) x(1-x)^{n}}{(n+\beta)^{2}\left(n^{2}-1\right)}\right]
\end{aligned}
$$

and hence

$$
\lim _{n \rightarrow \infty}\left\|B_{n}^{\alpha, \beta}\left(t^{2} ; x ; \lambda\right)-t^{2}\right\|_{C[0,1]}=0
$$

This implies $B_{n, \alpha, \beta}^{\lambda}(f ; x)$ converge uniformly to $f$ on $[0,1]$.

Recall that the first and second order Ditzian-Totik uniform modulus of smoothness are given by

$$
\omega_{\xi}(f, \delta):=\sup _{0<|h| \leq \delta} \sup _{x, x+h \xi(x) \in[0,1]}\{|f(x+h \xi(x))-f(x)|\}
$$

and

$$
\omega_{2}^{\phi}(f, \delta):=\sup _{0<|h| \leq \delta} \sup _{x, x \pm h \phi(x) \in[0,1]}\{|f(x+h \phi(x))-2 f(x)+f(x-h \phi(x))|\}
$$

respectively, where $\phi$ is an admissible step-weight function on $[a, b]$, that is, $\phi(x)=[(x-a)(b-x)]^{1 / 2}$ if $x \in[a, b]$ (see [24]). Let

$$
K_{2, \phi(x)}(f, \delta)=\inf _{g \in W^{2}(\phi)}\left\{\|f-g\|_{C[0,1]}+\delta\left\|\phi^{2} g^{\prime \prime}\right\|_{C[0,1]}: g \in C^{2}[0,1]\right\} \quad(\delta>0)
$$


be the corresponding $K$-functional, where

$$
W^{2}(\phi)=\left\{g \in C[0,1]: g^{\prime} \in A C[0,1], \phi^{2} g^{\prime \prime} \in C[0,1]\right\}
$$

and

$$
C^{2}[0,1]=\left\{g \in C[0,1]: g^{\prime}, g^{\prime \prime} \in C[0,1]\right\} .
$$

In this case, $g^{\prime} \in A C[0,1]$ means that $g^{\prime}$ is absolutely continuous on $[0,1]$. It is known by [25] that there exists an absolute constant $C>0$, such that

$$
C^{-1} \omega_{2}^{\phi}(f, \sqrt{\delta}) \leq K_{2, \phi(x)}(f, \delta) \leq C \omega_{2}^{\phi}(f, \sqrt{\delta}) .
$$

We are now ready to obtain global approximation theorem.

Theorem 2. Let $\lambda \in[-1,1]$ and $f \in C[0,1]$. Suppose that $\phi(\neq 0)$ such that $\phi^{2}$ is concave. Then

$$
\left|B_{n, \alpha, \beta}^{\lambda}(f ; x)-f(x)\right| \leq C \omega_{2}^{\phi}\left(f, \frac{\delta_{n}(\alpha, \beta, \lambda ; x)}{2 \phi(x)}\right)+\omega_{\xi}\left(f, \frac{\mu_{n}(\alpha, \beta, \lambda ; x)}{\xi(x)}\right)
$$

for $x \in[0,1]$ and $C>0$, where $\mu_{n}(\alpha, \beta, \lambda ; x)=B_{n, \alpha, \beta}^{\lambda}(t-x ; x), \delta_{n}(\alpha, \beta, \lambda ; x)=\left(v_{n}(\alpha, \beta, \lambda ; x)+\right.$ $\left.\mu_{n}^{2}(\alpha, \beta, \lambda ; x)(x)\right)^{\frac{1}{2}}$ and $v_{n}(\alpha, \beta, \lambda ; x)(x)=B_{n, \alpha, \beta}^{\lambda}\left((t-x)^{2} ; x\right)$.

Proof. Consider the operators

$$
\begin{aligned}
\tilde{B}_{n, \alpha, \beta}^{\lambda}(f ; x) & =B_{n, \alpha, \beta}^{\lambda}(f ; x)+f(x) \\
& -f\left(\frac{\alpha-\beta x}{n+\beta}+\lambda \frac{\alpha x(1-x)^{n}}{n+\beta}+\lambda \frac{1-2 x+x^{n+1}+(\alpha-1)(1-x)^{n+1}}{(n+\beta)(n-1)}\right)
\end{aligned}
$$

for $\lambda \in[-1,1], x \in[0,1]$. We observe that $\tilde{B}_{n, \alpha, \beta}^{\lambda}(1 ; x)=1$ and $\tilde{B}_{n, \alpha, \beta}^{\lambda}(t ; x)=x$, that is $\tilde{B}_{n, \alpha, \beta}^{\lambda}(t-x ; x)=0$.

Let $u=\rho x+(1-\rho) t, \rho \in[0,1]$. Since $\phi^{2}$ is concave on $[0,1]$, we have $\phi^{2}(u) \geq \rho \phi^{2}(x)+(1-$ $\rho) \phi^{2}(t)$ and hence

$$
\frac{|t-u|}{\phi^{2}(u)} \leq \frac{\rho|x-t|}{\rho \phi^{2}(x)+(1-\rho) \phi^{2}(t)} \leq \frac{|t-x|}{\phi^{2}(x)}
$$

So

$$
\begin{aligned}
\left|\tilde{B}_{n, \alpha, \beta}^{\lambda}(f ; x)-f(x)\right| & \leq\left|\tilde{B}_{n, \alpha, \beta}^{\lambda}(f-g ; x)\right|+\left|\tilde{B}_{n, \alpha, \beta}^{\lambda}(g ; x)-g(x)\right|+|f(x)-g(x)| \\
& \leq 4\|f-g\|_{C[0,1]}+\left|\tilde{B}_{n, \alpha, \beta}^{\lambda}(g ; x)-g(x)\right| .
\end{aligned}
$$

We obtain the following relations by applying the Taylor's formula:

$$
\begin{aligned}
& \left|\tilde{B}_{n, \alpha, \beta}^{\lambda}(g ; x)-g(x)\right| \\
& \leq B_{n, \alpha, \beta}^{\lambda}\left(\left|\int_{x}^{t}\right| t-u|| g^{\prime \prime}(u)|d u| ; x\right)+\left|\int_{x}^{x+\mu_{n}}\right| x+\mu_{n}(\alpha, \beta, \lambda ; x)-u|| g^{\prime \prime}(u)|d u| \\
& \leq\left\|\phi^{2} g^{\prime \prime}\right\|_{C[0,1]} B_{n, \alpha, \beta}^{\lambda}\left(\left|\int_{x}^{t} \frac{|t-u|}{\phi^{2}(u)} d u\right| ; x\right)+\left\|\phi^{2} g^{\prime \prime}\right\|_{C[0,1]}\left|\int_{x}^{x+\mu_{n}} \frac{\left|x+\mu_{n}(\alpha, \beta, \lambda ; x)-u\right|}{\phi^{2}(u)} d u\right| \\
& \leq \phi^{-2}(x)\left\|\phi^{2} g^{\prime \prime}\right\|_{C[0,1]} B_{n, \alpha, \beta}^{\lambda}\left((t-x)^{2} ; x\right)+\phi^{-2}(x)\left\|\phi^{2} g^{\prime \prime}\right\|_{C[0,1]} \beta_{n}^{2}(x) .
\end{aligned}
$$


By using the definition of $K$-functional together with (6) and the inequalities (9) and (10), we have

$$
\begin{aligned}
\left|\tilde{B}_{n, \alpha, \beta}^{\lambda}(f ; x)-f(x)\right| & \leq \phi^{-2}(x)\left\|\phi^{2} g^{\prime \prime}\right\|_{C[0,1]}\left(v_{n}(\alpha, \beta, \lambda ; x)+\mu_{n}^{2}(\alpha, \beta, \lambda ; x)\right)+4\|f-g\|_{C[0,1]} \\
& \leq C \omega_{2}^{\phi}\left(f, \frac{\left(v_{n}(\alpha, \beta, \lambda ; x)+\mu_{n}^{2}(\alpha, \beta, \lambda ; x)\right)^{\frac{1}{2}}}{2 \phi(x)}\right) .
\end{aligned}
$$

Also, by first order Ditzian-Totik uniform modulus of smoothness, we have

$$
\begin{aligned}
\left|f\left(x+\mu_{n}\right)-f(x)\right| & =\left|f\left(x+\xi(x) \frac{\mu_{n}(\alpha, \beta, \lambda ; x)}{\xi(x)}\right)-f(x)\right| \\
& \leq \omega_{\xi}\left(f, \frac{\mu_{n}(\alpha, \beta, \lambda ; x)}{\xi(x)}\right) .
\end{aligned}
$$

Therefore, the following inequalities hold:

$$
\begin{aligned}
\left|B_{n, \alpha, \beta}^{\lambda}(f ; x)-f(x)\right| & \leq\left|\tilde{B}_{n, \alpha, \beta}^{\lambda}(f ; x)-f(x)\right|+\left|f\left(x+\mu_{n}(\alpha, \beta, \lambda ; x)\right)-f(x)\right| \\
& \leq C \omega_{2}^{\phi}\left(f, \frac{\delta_{n}(\alpha, \beta, \lambda ; x)}{2 \phi(x)}\right)+\omega_{\xi}\left(f, \frac{\mu_{n}(\alpha, \beta, \lambda ; x)}{\xi(x)}\right),
\end{aligned}
$$

which completes the proof.

In order to obtain next result, we first recall some concepts and results concerning modulus of continuity and Peetre's K-functional. For $\delta>0$, the modulus of continuity $w(f, \delta)$ of $f \in C[a, b]$ is given by

$$
w(f, \delta):=\sup \{|f(x)-f(y)|: x, y \in[a, b],|x-y| \leq \delta\} .
$$

It is also well known that, for any $\delta>0$ and each $x \in[a, b]$,

$$
|f(x)-f(y)| \leq \omega(f, \delta)\left(\frac{|x-y|}{\delta}+1\right) .
$$

For $f \in C[0,1]$, the second-order modulus of smoothness is given by

$$
w_{2}(f, \sqrt{\delta}):=\sup _{0<h \leq \sqrt{\delta}} \sup _{x, x+2 h \in[0,1]}\{|f(x+2 h)-2 f(x+h)+f(x)|\},
$$

and the corresponding Peetre's K-functional [26] is

$$
K_{2}(f, \delta)=\inf \left\{\|f-g\|_{C[0,1]}+\delta\left\|g^{\prime \prime}\right\|_{C[0,1]}: g \in W^{2}[0,1]\right\},
$$

where

$$
W^{2}[0,1]=\left\{g \in C[0,1]: g^{\prime}, g^{\prime \prime} \in C[0,1]\right\} .
$$

It is well-known that the inequality

$$
K_{2}(f, \delta) \leq C w_{2}(f, \sqrt{\delta}) \quad(\delta>0)
$$

holds in which the absolute constant $C>0$ is independent of $\delta$ and $f$ (see [25]).

We are now ready to establish a direct local approximation theorem for operators $B_{n, \alpha, \beta}^{\lambda}(f ; x)$ via second order modulus of smoothness and usual modulus of continuity. 
Theorem 3. Assume that $f \in C[0,1]$ and $x \in[0,1]$. Then there exists an absolute constant $C$ such that

$$
\left|B_{n, \alpha, \beta}^{\lambda}(f ; x)-f(x)\right| \leq C w_{2}\left(f, \frac{1}{2} \delta_{n}(\alpha, \beta, \lambda ; x)\right)+w\left(f, \mu_{n}(\alpha, \beta, \lambda ; x)\right)
$$

for the operators $B_{n, \alpha, \beta}^{\lambda}(f ; x)$, where $\mu_{n}(\alpha, \beta, \lambda ; x)$ and $\delta_{n}(\alpha, \beta, \lambda ; x)$ are given in Theorem 2 .

Proof. Consider the operators $\tilde{B}_{n, \alpha, \beta}^{\lambda}(f ; x)$ as defined in Theorem 2. Assume that $t, x \in[0,1]$ and $g \in W^{2}[0,1]$. The following equality yields by Taylor's expansion formula:

$$
g(t)=g(x)+(t-x) g^{\prime}(x)+\int_{x}^{t}(t-u) g^{\prime \prime}(u) d u .
$$

If we apply $\tilde{B}_{n, \alpha, \beta}^{\lambda}(\cdot ; x)$ to both sides of (13) and keeping in mind these operators preserve constants and linear functions, we obtain

$$
\begin{aligned}
\tilde{B}_{n, \alpha, \beta}^{\lambda}(g ; x)-g(x) & =g^{\prime}(x) \tilde{B}_{n, \alpha, \beta}^{\lambda}(t-x ; x)+\tilde{B}_{n, \alpha, \beta}^{\lambda}\left(\int_{x}^{t}(t-u) g^{\prime \prime}(u) d u ; x\right) \\
& =B_{n, \alpha, \beta}^{\lambda}\left(\int_{x}^{t}(t-u) g^{\prime \prime}(u) d u ; x\right)-\int_{x}^{x+\mu_{n}}\left(x+\mu_{n}(\alpha, \beta, \lambda ; x)-u\right) g^{\prime \prime}(u) d u .
\end{aligned}
$$

Therefore,

$$
\begin{aligned}
\left|\tilde{B}_{n, \alpha, \beta}^{\lambda}(g ; x)-g(x)\right| & \leq B_{n, \alpha, \beta}^{\lambda}\left(\left|\int_{x}^{t}\right| t-u|| g^{\prime \prime}(u)|d u| ; x\right) \\
& -\int_{x}^{x+\mu_{n}}\left|x+\mu_{n}(\alpha, \beta, \lambda ; x)-u\right|\left|g^{\prime \prime}(u)\right| d u \\
& \leq\left\|g^{\prime \prime}\right\|_{C[0,1]}\left(B_{n, \alpha, \beta}^{\lambda}\left((t-x)^{2} ; x\right)+\left(B_{n, \alpha, \beta}^{\lambda}(t-x ; x)\right)^{2}\right) .
\end{aligned}
$$

With the help of (7), one obtains

$$
\begin{aligned}
\left\|\tilde{B}_{n, \alpha, \beta}^{\lambda}(g ; x)\right\|_{C[0,1]} & \leq\left\|B_{n, \alpha, \beta}^{\lambda}(g ; x)\right\|_{C[0,1]}+\|g(x)\|_{C[0,1]}+\left\|g\left(x+\mu_{n}(\alpha, \beta, \lambda ; x)\right)\right\|_{C[0,1]} \\
& \leq\|3 g\|_{C[0,1]} .
\end{aligned}
$$

Now, for $f \in C[0,1]$ and $g \in W^{2}[0,1]$, using (7) and (14), we get

$$
\begin{aligned}
\left|B_{n, \alpha, \beta}^{\lambda}(f ; x)-f(x)\right| \leq & \left|\tilde{B}_{n, \alpha, \beta}^{\lambda}(f-g ; x)\right|+\left|\tilde{B}_{n, \alpha, \beta}^{\lambda}(g ; x)-g(x)\right| \\
& +|g(x)-f(x)|+\left|f\left(x+\mu_{n}(\alpha, \beta, \lambda ; x)\right)-f(x)\right| \\
\leq & \delta_{n}^{2}(\alpha, \beta, \lambda ; x)\left\|g^{\prime \prime}\right\|_{C[0,1]}+w\left(f, \mu_{n}(\alpha, \beta, \lambda ; x)\right)+4\|f-g\|_{C[0,1]} .
\end{aligned}
$$

Finally, by assuming the infimum on the right-hand side of the above inequality over all $g \in W^{2}[0,1]$ togrther with inequality (12), we obtain

$$
\begin{aligned}
\left|B_{n, \alpha, \beta}^{\lambda}(f ; x)-f(x)\right| & \leq 4 K_{2}\left(f, \frac{\delta_{n}^{2}(\alpha, \beta, \lambda ; x)}{4}\right)+w\left(f, \mu_{n}(\alpha, \beta, \lambda ; x)\right) \\
& \leq C w_{2}\left(f, \frac{1}{2} \delta_{n}(\alpha, \beta, \lambda ; x)\right)+w\left(f, \mu_{n}(\alpha, \beta, \lambda ; x)\right),
\end{aligned}
$$

which completes the proof. 
In the following theorem, we obtain a local direct estimate of the rate of convergence via Lipschitz-type function involving two parameters for the operators $B_{n, \alpha, \beta}^{\lambda}$. Before proceeding further, let us recall that

$$
\operatorname{Lip}_{M}^{\left(k_{1}, k_{2}\right)}(\eta):=\left\{f \in C[0,1]:|f(t)-f(x)| \leq M \frac{|t-x|^{\eta}}{\left(k_{1} x^{2}+k_{2} x+t\right)^{\frac{\eta}{2}}} ; x \in(0,1], t \in[0,1]\right\}
$$

for $k_{1} \geq 0, k_{2}>0$, where $\eta \in(0,1]$ and $M$ is a positive constant (see [27]).

Theorem 4. If $f \in \operatorname{Lip}_{M}^{\left(k_{1}, k_{2}\right)}(\eta)$, then

$$
\left|B_{n, \alpha, \beta}^{\lambda}(f ; x)-f(x)\right| \leq M \sqrt{\frac{v_{n}^{\eta}(\alpha, \beta, \lambda ; x)}{\left(k_{1} x^{2}+k_{2} x\right)^{\eta}}}
$$

for all $\lambda \in[-1,1], x \in(0,1]$ and $\eta \in(0,1]$, where $v_{n}(\alpha, \beta, \lambda ; x)$ is defined in Theorem 2 .

Proof. Let $f \in \operatorname{Lip}_{M}^{\left(k_{1}, k_{2}\right)}(\eta)$ and $\eta \in(0,1]$. First, we are going to show that statement is true for $\eta=1$. We write

$$
\begin{aligned}
\left|B_{n, \alpha, \beta}^{\lambda}(f ; x)-f(x)\right| & \leq\left|B_{n, \alpha, \beta}^{\lambda}(|f(t)-f(x)| ; x)\right|+f(x)\left|B_{n, \alpha, \beta}^{\lambda}(1 ; x)-1\right| \\
& \leq \sum_{i=0}^{n}\left|f\left(\frac{i+\alpha}{n+\beta}\right)-f(x)\right| \tilde{b}_{n, i}(x ; \lambda) \\
& \leq M \sum_{i=0}^{n} \frac{\left|\frac{i+\alpha}{n+\beta}-x\right|}{\left(k_{1} x^{2}+k_{2} x+t\right)^{\frac{1}{2}}} \tilde{b}_{n, i}(x ; \lambda)
\end{aligned}
$$

for $f \in \operatorname{Lip}_{M}^{\left(k_{1}, k_{2}\right)}(1)$. By using the relation

$$
\left(k_{1} x^{2}+k_{2} x+t\right)^{-1 / 2} \leq\left(k_{1} x^{2}+k_{2} x\right)^{-1 / 2} \quad\left(k_{1} \geq 0, k_{2}>0\right)
$$

and applying Cauchy-Schwarz inequality, we obtain

$$
\begin{aligned}
\left|B_{n, \alpha, \beta}^{\lambda}(f ; x)-f(x)\right| & \leq M\left(k_{1} x^{2}+k_{2} x\right)^{-1 / 2} \sum_{i=0}^{n}\left|\frac{i+\alpha}{n+\beta}-x\right| \tilde{b}_{n, i}(x ; \lambda) \\
& =M\left(k_{1} x^{2}+k_{2} x\right)^{-1 / 2}\left|B_{n, \alpha, \beta}^{\lambda}(t-x ; x)\right| \\
& \leq M\left|v_{n}(\alpha, \beta, \lambda ; x)\right|^{1 / 2}\left(k_{1} x^{2}+k_{2} x\right)^{-1 / 2} .
\end{aligned}
$$

Hence, the statement is true for $\eta=1$. By the monotonicity of $B_{n, \alpha, \beta}^{\lambda}(f ; x)$ and applying Hölder's inequality two times with $a=2 / \eta$ and $b=2 /(2-\eta)$, we can see that the statement is true for $\eta \in(0,1]$ as follows:

$$
\begin{aligned}
\left|B_{n, \alpha, \beta}^{\lambda}(f ; x)-f(x)\right| & \leq \sum_{i=0}^{n}\left|f\left(\frac{i+\alpha}{n+\beta}\right)-f(x)\right| \tilde{b}_{n, i}(x ; \lambda) \\
& \leq\left(\sum_{i=0}^{n}\left|f\left(\frac{i+\alpha}{n+\beta}\right)-f(x)\right|^{\frac{2}{\eta}} \tilde{b}_{n, i}(x ; \lambda)\right)^{\frac{\eta}{2}}\left(\sum_{i=0}^{n} \tilde{b}_{n, i}(x ; \lambda)\right)^{\frac{2-\eta}{2}} \\
& \leq M\left(\sum_{i=0}^{n} \frac{\left(\frac{i+\alpha}{n+\beta}-x\right)^{2} \tilde{b}_{n, i}(x ; \lambda)}{\frac{i+\alpha}{n+\beta}+k_{1} x^{2}+k_{2} x}\right)^{\frac{\eta}{2}}
\end{aligned}
$$




$$
\begin{aligned}
& \leq M\left(k_{1} x^{2}+k_{2} x\right)^{-\eta / 2}\left\{\sum_{i=0}^{n}\left(\frac{i+\alpha}{n+\beta}-x\right)^{2} \tilde{b}_{n, i}(x ; \lambda)\right\}^{\frac{\eta}{2}} \\
& \leq M\left(k_{1} x^{2}+k_{2} x+t\right)^{-\eta / 2}\left[B_{n}^{\alpha, \beta}\left((t-x)^{2} ; x ; \lambda\right)\right]^{\frac{\eta}{2}} \\
& =M \sqrt{\frac{v_{n}^{\eta}(\alpha, \beta, \lambda ; x)}{\left(k_{1} x^{2}+k_{2} x\right)^{\eta}}}
\end{aligned}
$$

Theorem 5. The following inequality holds:

$$
\left|B_{n, \alpha, \beta}^{\lambda}(f ; x)-f(x)\right| \leq\left|\mu_{n}(\alpha, \beta, \lambda ; x)\right|\left|f^{\prime}(x)\right|+2 \sqrt{v_{n}(\alpha, \beta, \lambda ; x)} w\left(f^{\prime}, \sqrt{v_{n}(\alpha, \beta, \lambda ; x)}\right)
$$

for $f \in C^{1}[0,1]$ and $x \in[0,1]$, where $\mu_{n}(\alpha, \beta, \lambda ; x)$ and $\nu_{n}(\alpha, \beta, \lambda ; x)$ are defined in Theorem 2 .

Proof. We have

$$
f(t)-f(x)=(t-x) f^{\prime}(x)+\int_{x}^{t}\left(f^{\prime}(u)-f^{\prime}(x)\right) d u
$$

for any $t \in[0,1]$ and $x \in[0,1]$. By applying the operators $B_{n, \alpha, \beta}^{\lambda}(\cdot ; x)$ to both sides of $(15)$, we have

$$
B_{n, \alpha, \beta}^{\lambda}(f(t)-f(x) ; x)=f^{\prime}(x) B_{n, \alpha, \beta}^{\lambda}(t-x ; x)+B_{n, \alpha, \beta}^{\lambda}\left(\int_{x}^{t}\left(f^{\prime}(u)-f^{\prime}(x)\right) d u ; x\right) .
$$

The following inequality holds for any $\delta>0, u \in[0,1]$ and $f \in C[0,1]$ :

$$
|f(u)-f(x)| \leq w(f, \delta)\left(\frac{|u-x|}{\delta}+1\right) .
$$

Thus, we obtain

$$
\left|\int_{x}^{t}\left(f^{\prime}(u)-f^{\prime}(x)\right) d u\right| \leq w\left(f^{\prime}, \delta\right)\left(\frac{(t-x)^{2}}{\delta}+|t-x|\right)
$$

Hence

$$
\begin{aligned}
\left|B_{n, \alpha, \beta}^{\lambda}(f ; x)-f(x)\right| & \leq\left|f^{\prime}(x)\right|\left|B_{n, \alpha, \beta}^{\lambda}(t-x ; x)\right| \\
& +w\left(f^{\prime}, \delta\right)\left\{\frac{1}{\delta} B_{n, \alpha, \beta}^{\lambda}\left((t-x)^{2} ; x\right)+B_{n, \alpha, \beta}^{\lambda}(t-x ; x)\right\} .
\end{aligned}
$$

By applying Cauchy-Schwarz inequality on the right hand side of last inequality (16), we have

$$
\begin{aligned}
\left|B_{n, \alpha, \beta}^{\lambda}(f ; x)-f(x)\right| & \leq\left|f^{\prime}(x)\right|\left|\mu_{n}(\alpha, \beta, \lambda ; x)\right| \\
& +w\left(f^{\prime}, \delta\right)\left\{\frac{1}{\delta} \sqrt{B_{n, \alpha, \beta}^{\lambda}\left((t-x)^{2} ; x\right)}+1\right\} \sqrt{B_{n, \alpha, \beta}^{\lambda}(|t-x| ; x)} .
\end{aligned}
$$

Consequently, we obtain the desired result if we choose $\delta$ as $v_{n}^{1 / 2}(\alpha, \beta, \lambda ; x)$.

\section{Voronovskaja-Type Theorems}

Here, we prove the following Voronovskaja-type theorems by $B_{n, \alpha, \beta}^{\lambda}(f ; x)$. 
Theorem 6. Let $f, f^{\prime}, f^{\prime \prime} \in C_{B}[0,1]$, where $C_{B}[0,1]$ is the set of all real-valued bounded and continuous functions defined on $[0,1]$. Then, for each $x \in[0,1]$, we have

$$
\lim _{n \rightarrow \infty} n\left\{B_{n, \alpha, \beta}^{\lambda}(f ; x)-f(x)\right\}=(\alpha-\beta x) f^{\prime}(x)+\frac{x(1-x)}{2} f^{\prime \prime}(x)
$$

uniformly on $[0,1]$.

Proof. We first write the following equality by Taylor's expansion theorem of function $f(x)$ in $C_{B}[0,1]$ :

$$
f(t)=f(x)+(t-x) f^{\prime}(x)+\frac{1}{2}(t-x)^{2} f^{\prime \prime}(x)+(t-x)^{2} r_{x}(t),
$$

where $r_{x}(t)$ is Peano form of the remainder, $r_{x} \in C[0,1]$ and $r_{x}(t) \rightarrow 0$ as $t \rightarrow x$. Applying the operators $B_{n, \alpha, \beta}^{\lambda}(\cdot ; x)$ to identity (17), we have

$$
B_{n, \alpha, \beta}^{\lambda}(f ; x)-f(x)=f^{\prime}(x) B_{n, \alpha, \beta}^{\lambda}(t-x ; x)+\frac{f^{\prime \prime}(x)}{2} B_{n, \alpha, \beta}^{\lambda}\left((t-x)^{2} ; x\right)+B_{n, \alpha, \beta}^{\lambda}\left((t-x)^{2} r_{x}(t) ; x\right) .
$$

Using Cauchy-Schwarz inequality, we have

$$
B_{n, \alpha, \beta}^{\lambda}\left((t-x)^{2} r_{x}(t) ; x\right) \leq \sqrt{B_{n, \alpha, \beta}^{\lambda}\left((t-x)^{4} ; x\right)} \sqrt{B_{n, \alpha, \beta}^{\lambda}\left(r_{x}^{2}(t) ; x\right)} .
$$

We observe that $\lim _{n} B_{n, \alpha, \beta}^{\lambda}\left(r_{x}^{2}(t) ; x\right)=0$ and hence

$$
\lim _{n \rightarrow \infty} n\left\{B_{n, \alpha, \beta}^{\lambda}\left((t-x)^{2} r_{x}(t) ; x\right)\right\}=0 .
$$

Thus

$$
\begin{aligned}
\lim _{n \rightarrow \infty} n\left\{B_{n, \alpha, \beta}^{\lambda}(f ; x)-f(x)\right\}= & \lim _{n \rightarrow \infty} n\left\{B_{n, \alpha, \beta}^{\lambda}(t-x ; x) f^{\prime}(x)+\frac{f^{\prime \prime}(x)}{2} B_{n, \alpha, \beta}^{\lambda}\left((t-x)^{2} ; x\right)\right. \\
& \left.+B_{n, \alpha, \beta}^{\lambda}\left((t-x)^{2} r_{x}(t) ; x\right)\right\} .
\end{aligned}
$$

The result follows immediately by applying the Corollaries 1 and 2 .

For $f \in C[0,1]$ and $\delta>0$, the Ditzian-Totik modulus of smoothness is given by

$$
\omega_{\phi}(f, \delta):=\sup _{0<|h| \leq \delta}\left\{\left|f\left(x+\frac{h \phi(x)}{2}\right)-f\left(x-\frac{h \phi(x)}{2}\right)\right|, x \pm \frac{h \phi(x)}{2} \in[0,1]\right\},
$$

where $\phi(x)=(x(1-x))^{1 / 2}$, and let

$$
K_{\phi}(f, \delta)=\inf _{g \in W_{\phi}[0,1]}\left\{\|f-g\|+\delta\left\|\phi g^{\prime}\right\|: g \in C^{1}[0,1]\right\}
$$

be the corresponding Peetre's K-functional, where

$$
W_{\phi}[0,1]=\left\{g: g \in A C_{l o c}[0,1],\left\|\phi g^{\prime}\right\|<\infty\right\}
$$

and $A C_{l o c}[0,1]$ denotes the class of absolutely continuous functions defined on $[a, b] \subset[0,1]$. There exists a constant $C>0$ such that $K_{\phi}(f, \delta) \leq C \omega_{\phi}(f, \delta)$.

Next, we give a quantitative Voronovskaja-type result for $B_{n}^{\alpha, \beta}(f ; x ; \lambda)$. 
Theorem 7. Suppose that $f \in C[0,1]$ such that $f^{\prime}, f^{\prime \prime} \in C[0,1]$. Then

$$
\begin{aligned}
& \left|B_{n, \alpha, \beta}^{\lambda}(f ; x) f(x)-f(x)-\mu_{n}(\alpha, \beta, \lambda ; x) f^{\prime}(x)-\left\{v_{n}(\alpha, \beta, \lambda ; x)+1\right\} \frac{f^{\prime \prime}(x)}{2}\right| \\
& \quad \leq \frac{C}{n} \phi^{2}(x) \omega_{\phi}\left(f^{\prime \prime}, \frac{1}{\sqrt{n}}\right) .
\end{aligned}
$$

for every $x \in[0,1]$ and sufficiently large $n$, where $C$ is a positive constant, $\mu_{n}(\alpha, \beta, \lambda ; x)$ and $v_{n}(\alpha, \beta, \lambda ; x)$ are defined in Theorem 2.

Proof. Consider the following equality

$$
f(t)-f(x)-(t-x) f^{\prime}(x)=\int_{x}^{t}(t-u) f^{\prime \prime}(u) d u
$$

for $f \in C[0,1]$. It follows that

$$
f(t)-f(x)-(t-x) f^{\prime}(x)-\frac{f^{\prime \prime}(x)}{2}\left((t-x)^{2}+1\right) \leq \int_{x}^{t}(t-u)\left[f^{\prime \prime}(u)-f^{\prime \prime}(x)\right] d u .
$$

Applying $B_{n, \alpha, \beta}^{\lambda}(\cdot ; x)$ to both sides of (20), we obtain

$$
\begin{aligned}
& \left|B_{n, \alpha, \beta}^{\lambda}(f ; x)-f(x)-B_{n, \alpha, \beta}^{\lambda}((t-x) ; x) f^{\prime}(x)-\frac{f^{\prime \prime}(x)}{2}\left(B_{n, \alpha, \beta}^{\lambda}\left((t-x)^{2} ; x\right)+B_{n, \alpha, \beta}^{\lambda}(1 ; x)\right)\right| \\
& \leq B_{n, \alpha, \beta}^{\lambda}\left(\left|\int_{x}^{t}\right| t-u|| f^{\prime \prime}(u)-f^{\prime \prime}(x)|d u| ; x\right) .
\end{aligned}
$$

The quantity in the right hand side of (21) can be estimated as

$$
\left|\int_{x}^{t}\right| t-u|| f^{\prime \prime}(u)-f^{\prime \prime}(x)|d u| \leq 2\left\|f^{\prime \prime}-g\right\|(t-x)^{2}+2\left\|\phi g^{\prime}\right\| \phi^{-1}(x)|t-x|^{3},
$$

where $g \in W_{\phi}[0,1]$. There exists $C>0$ such that

$$
B_{n, \alpha, \beta}^{\lambda}\left((t-x)^{2} ; x\right) \leq \frac{C}{2 n} \phi^{2}(x) \quad \text { and } \quad B_{n, \alpha, \beta}^{\lambda}\left((t-x)^{4} ; x\right) \leq \frac{C}{2 n^{2}} \phi^{4}(x)
$$

for sufficiently large $n$. By taking (21)-(23) into our account and using Cauchy-Schwarz inequality, we have

$$
\begin{aligned}
& \left|B_{n, \alpha, \beta}^{\lambda}(f ; x)-f(x)-B_{n, \alpha, \beta}^{\lambda}((t-x) ; x) f^{\prime}(x)-\frac{f^{\prime \prime}(x)}{2}\left(B_{n, \alpha, \beta}^{\lambda}\left((t-x)^{2} ; x\right)+B_{n, \alpha, \beta}^{\lambda}(1 ; x)\right)\right| \\
& \leq 2\left\|f^{\prime \prime}-g\right\| B_{n, \alpha, \beta}^{\lambda}\left((t-x)^{2} ; x\right)+2\left\|\phi g^{\prime}\right\| \phi^{-1}(x) B_{n, \alpha, \beta}^{\lambda}\left(|t-x|^{3} ; x\right) \\
& \leq \frac{C}{n} x(1-x)\left\|f^{\prime \prime}-g\right\|+2\left\|\phi g^{\prime}\right\| \phi^{-1}(x)\left\{B_{n, \alpha, \beta}^{\lambda}\left((t-x)^{2} ; x\right)\right\}^{1 / 2}\left\{B_{n, \alpha, \beta}^{\lambda}\left((t-x)^{4} ; x\right)\right\}^{1 / 2} \\
& \leq \frac{C}{n} \phi^{2}(x)\left\{\left\|f^{\prime \prime}-g\right\|+n^{-1 / 2}\left\|\phi g^{\prime}\right\|\right\} .
\end{aligned}
$$

Finally, by taking infimum over all $g \in W_{\phi}[0,1]$, this last inequality leads us to the assertion (19) of Theorem 7.

As an immediate consequence of Theorem 7, we have the following result. 
Corollary 3. If $f \in C[0,1]$ such that $f^{\prime}, f^{\prime \prime} \in C[0,1]$, then

$$
\lim _{n \rightarrow \infty} n\left|B_{n, \alpha, \beta}^{\lambda}(f ; x) f(x)-f(x)-\mu_{n}(\alpha, \beta, \lambda ; x) f^{\prime}(x)-\left\{v_{n}(\alpha, \beta, \lambda ; x)+1\right\} \frac{f^{\prime \prime}(x)}{2}\right|=0,
$$

where $\mu_{n}(\alpha, \beta, \lambda ; x)$ and $v_{n}(\alpha, \beta, \lambda ; x)$ are defined in Theorem 2.

\section{The Bivariate Case of the Operators $B_{n, \alpha, \beta}^{\lambda}(f ; x)$}

We construct bivariate version of Stancu-type $\lambda$-Bernstein operators defined which was defined in the first section of this manuscript as (5) and study their approximation properties.

For $0 \leq \alpha_{i} \leq \beta_{i}(i=1,2)$, we defined the bivariate version of Stancu-type $\lambda$-Bernstein operators by

$$
B_{n, m}^{\lambda, \alpha, \beta}(f ; x, y)=\sum_{i_{1}=0}^{n} \sum_{i_{2}=0}^{m} f\left(\frac{i_{1}+\alpha_{1}}{n+\beta_{1}}, \frac{i_{2}+\alpha_{2}}{m+\beta_{2}}\right) \tilde{b}_{n, i_{1}}\left(\lambda_{1} ; x\right) \tilde{b}_{m, i_{2}}\left(\lambda_{2} ; y\right)
$$

for $(x, y) \in I$ and $f \in C(I)$, where $I=[0,1] \times[0,1]$ and $\tilde{b}_{n, i_{1}}\left(\lambda_{1} ; x\right)$ and $\tilde{b}_{m, i_{2}}\left(\lambda_{2} ; x\right)$ are Bézier bases defined in (4).

We remark that if we take $\lambda_{1}=\lambda_{2}=0$ in bivariate $\lambda$-Bernstein-Stancu operators, then (24) reduces to the classical bivariate Bernstein-Stancu operators defined in [28]. Also, for $\alpha_{1}=\beta_{1}=\lambda_{1}=0$ and $\alpha_{2}=\beta_{2}=\lambda_{2}=0$, the bivariate $\lambda$-Bernstein-Stancu operators (24) reduce to classical bivariate Bernstein operators defined in [29].

Lemma 2. The following equalities hold for bivariate $\lambda$-Bernstein-Stancu operators:

$$
\begin{aligned}
B_{n, m}^{\lambda, \alpha, \beta}(1 ; x, y)= & 1 ; \\
B_{n, m}^{\alpha, \beta}(s ; x, y)= & \frac{\alpha_{1}+n x}{n+\beta_{1}}+\lambda_{1}\left[\frac{1-2 x+x^{n+1}+\left(\alpha_{1}-1\right)(1-x)^{n+1}}{\left(n+\beta_{1}\right)(n-1)}+\frac{\alpha_{1} x(1-x)^{n}}{n+\beta_{1}}\right] ; \\
B_{n, m}^{\lambda, \alpha, \beta}(t ; x, y)= & \frac{\alpha_{2}+m y}{m+\beta_{2}}+\lambda_{2}\left[\frac{1-2 y+y^{m+1}+\left(\alpha_{2}-1\right)(1-y)^{m+1}}{\left(m+\beta_{2}\right)(m-1)}+\frac{\alpha_{2} y(1-y)^{m}}{m+\beta_{2}}\right] ; \\
B_{n, m}^{\lambda, \alpha, \beta}\left(s^{2} ; x, y\right)= & \frac{1}{\left(n+\beta_{1}\right)^{2}}\left\{n(n-1) x^{2}+\left(1+2 \alpha_{1}\right) n x+\alpha_{1}^{2}\right\} \\
& +\lambda_{1}\left[\frac{2 n x-1-4 n x^{2}+(2 n+1) x^{n+1}+(1-x)^{n+1}}{\left(n+\beta_{1}\right)^{2}(n-1)}+\frac{\alpha_{1}^{2}-4 \alpha_{1} x}{\left(n+\beta_{1}\right)^{2}(n-1)}\right. \\
& \left.+\frac{2 \alpha_{1} n-2 \alpha_{1}\left(\alpha_{1}+n\right)\left(x^{n+1}+(1-x)^{n}\right)+\alpha_{1}^{2} x\left(n^{2}+1\right)(1-x)^{n}}{\left(n+\beta_{1}\right)^{2}\left(n^{2}-1\right)}\right] \\
B_{n, m}^{\lambda, \alpha, \beta}\left(t^{2} ; x, y\right)= & \frac{1}{\left(m+\beta_{2}\right)^{2}}\left\{m(m-1) y^{2}+\left(1+2 \alpha_{2}\right) m y+\alpha_{2}^{2}\right\} \\
& +\lambda_{2}\left[\frac{2 m y-1-4 m y^{2}+(2 m+1) y^{m+1}+(1-y)^{m+1}}{\left(m+\beta_{2}\right)^{2}(m-1)}+\frac{\alpha_{2}^{2}-4 \alpha_{2} y}{\left(n+\beta_{2}\right)^{2}(m-1)}\right. \\
& \left.+\frac{2 \alpha_{2} m-2 \alpha_{2}\left(\alpha_{2}+m\right)\left(y^{m+1}+(1-y)^{m}\right)+\alpha_{2}^{2} y\left(m^{2}+1\right)(1-y)^{m}}{\left(m+\beta_{2}\right)^{2}\left(m^{2}-1\right)}\right] .
\end{aligned}
$$

Theorem 8. Let $e_{i j}(x, y)=x^{i} y^{j}$, where $0 \leq i+j \leq 2$. Then, the sequence $B_{n, m}^{\lambda, \alpha, \beta}(f ; x, y)$ of operators converges uniformly to $f$ on I for each $f \in C(I)$.

Proof. It is enough to prove the following condition

$$
\lim _{n, m \rightarrow \infty} B_{n, m}^{\lambda, \alpha, \beta}\left(e_{i j} ; x, y\right)=e_{i j}
$$


converges uniformly on $I$. With the help of Lemma 2, one can see that

$$
\begin{gathered}
\lim _{m, n \rightarrow \infty} B_{n, m}^{\lambda, \alpha, \beta}\left(e_{00} ; x, y\right)=e_{00}, \\
\lim _{n, m \rightarrow \infty} B_{n, m}^{\lambda, \alpha, \beta}\left(e_{10} ; x, y\right)=e_{10}, \quad \lim _{n, m \rightarrow \infty} B_{n, m}^{\lambda, \alpha, \beta}\left(e_{01} ; x, y\right)=e_{01}
\end{gathered}
$$

and

$$
\lim _{n, m \rightarrow \infty} B_{n, m}^{\lambda, \alpha, \beta}\left(e_{02}+e_{20} ; x, y\right)=e_{02}+e_{20} .
$$

Keeping in mind the above conditions and Korovkin type theorem established by Volkov [30], we obtain

$$
\lim _{m, n \rightarrow \infty} B_{n, m}^{\lambda, \alpha, \beta}(f ; x, y)=f
$$

converges uniformly.

Now, we compute the rate of convergence of operators (24) by means of the modulus of continuity. Recall that the modulus of continuity for bivariate case is defined as

$$
\omega(f, \delta)=\sup \left\{|f(s, t)-f(x, y)|: \sqrt{(s-x)^{2}+(t-y)^{2}} \leq \delta\right\}
$$

for $f \in C\left(I_{a b}\right)$ and for every $(s, t),(x, y) \in I_{a b}=[0, a] \times[0, b]$. The partial moduli of continuity with respect to $x$ and $y$ are defined by

$$
\begin{aligned}
& \omega_{1}(f, \delta)=\sup \left\{\left|f\left(x_{1}, y\right)-f\left(x_{2}, y\right)\right|: y \in[0, a] \text { and }\left|x_{1}-x_{2}\right| \leq \delta\right\} \\
& \omega_{2}(f, \delta)=\sup \left\{\left|f\left(x, y_{1}\right)-f\left(x, y_{2}\right)\right|: x \in[0, b] \text { and }\left|y_{1}-y_{2}\right| \leq \delta\right\}
\end{aligned}
$$

Peetre's $K$-functional is given by

$$
K(f, \delta)=\inf _{g \in C^{2}\left(I_{a b}\right)}\left\{\|f-g\|_{C\left(I_{a b}\right)}+\delta\|g\|_{C^{2}\left(I_{a b}\right)}\right\}
$$

for $\delta>0$, where $C^{2}\left(I_{a b}\right)$ is the space of functions of $f$ such that $f, \frac{\partial^{j} f}{\partial x^{j}}$ and $\frac{\partial^{j} f}{\partial y^{j}}(j=1,2)$ in $C\left(I_{a b}\right)$ [26]. We now give an estimate of the rates of convergence of operators $B_{n, m}^{\lambda, \alpha, \beta}(f ; x, y)$.

Theorem 9. Let $f \in C(I)$. Then

$$
\left|B_{n, m}^{\lambda, \alpha, \beta}(f ; x, y)-f(x, y)\right| \leq 4 \omega\left(f ; v_{n}^{1 / 2}(\alpha, \beta, \lambda ; x), v_{m}^{1 / 2}(\alpha, \beta, \lambda ; y)\right)
$$

for all $x \in I$, where

$$
v_{n}(\alpha, \beta, \lambda ; x)=B_{n, m}^{\lambda, \alpha, \beta}\left((s-x)^{2} ; x, y\right) \text { and } v_{m}(\alpha, \beta, \lambda ; y)=B_{n, m}^{\lambda, \alpha, \beta}\left((t-y)^{2} ; x, y\right) .
$$

Proof. Since (24) is linear and positive, we have

$$
\begin{aligned}
\left|B_{n, m}^{\lambda, \alpha, \beta}(f ; x, y)-f(x, y)\right| & \leq B_{n, m}^{\lambda, \alpha, \beta}(|f(s, t)-f(x, y)| ; x, y) \\
\leq & B_{n, m}^{\lambda, \alpha, \beta}\left(\omega\left(f ; \sqrt{(s-x)^{2}+(t-y)^{2}}\right) ; x, y\right) \\
\leq & \omega\left(f ; \sqrt{v_{n}(\alpha, \beta, \lambda ; x)}, \sqrt{v_{m}(\alpha, \beta, \lambda ; y)}\right)
\end{aligned}
$$




$$
\times\left[\frac{1}{\sqrt{v_{n}(\alpha, \beta, \lambda ; x) v_{m}(\alpha, \beta, \lambda ; y)}} B_{n, m}^{\lambda, \alpha, \beta}\left(\sqrt{(s-x)^{2}+(t-y)^{2}} ; x, y\right)\right] .
$$

The Cauchy-Schwartz inequality gives that

$$
\begin{aligned}
& \left|B_{n, m}^{\lambda, \alpha, \beta}(f ; x, y)-f(x, y)\right| \\
& \leq \omega\left(f ; \sqrt{v_{n}(\alpha, \beta, \lambda ; x)}, \sqrt{v_{m}(\alpha, \beta, \lambda ; y)}\right) \\
& \times\left[1+\frac{1}{\sqrt{v_{n}(\alpha, \beta, \lambda ; x) v_{m}(\alpha, \beta, \lambda ; y)}}\left\{B_{n, m}^{\lambda, \alpha, \beta}\left((s-x)^{2} ; x, y\right) B_{n, m}^{\lambda, \alpha, \beta}\left((t-y)^{2} ; x, y\right)\right\}^{1 / 2}\right. \\
& \left.+\frac{\sqrt{B_{n, m}^{\lambda, \alpha, \beta}\left((s-x)^{2} ; x, y\right)}}{\sqrt{v_{n}(\alpha, \beta, \lambda ; x)}}+\frac{\sqrt{B_{n, m}^{\lambda, \alpha, \beta}\left((t-y)^{2} ; x, y\right)}}{\sqrt{v_{m}(\alpha, \beta, \lambda ; y)}}\right] .
\end{aligned}
$$

If we choose

$$
v_{n}(\alpha, \beta, \lambda ; x)=B_{n, m}^{\lambda, \alpha, \beta}\left((s-x)^{2} ; x, y\right) \quad \text { and } \quad v_{m}(\alpha, \beta, \lambda ; y)=B_{n, m}^{\lambda, \alpha, \beta}\left((t-y)^{2} ; x, y\right)
$$

for all $(x, y) \in I$ we complete the proof, where

$$
\begin{aligned}
B_{n, m}^{\lambda, \alpha, \beta}\left((s-x)^{2} ; x, y\right)= & B_{n, m}^{\lambda, \alpha, \beta}\left(s^{2} ; x, y\right)-2 x B_{n, m}^{\lambda, \alpha, \beta}(s ; x, y)+x^{2} B_{n, m}^{\lambda, \alpha, \beta}(1 ; x, y) \\
= & \frac{n x(1-x)+\left(\beta_{1} x-\alpha_{1}\right)^{2}}{\left(n+\beta_{1}\right)^{2}} \\
& +\lambda_{1}\left[\frac{4 x^{2}-2 x-2 x^{n+2}-2\left(\alpha_{1}-1\right) x(1-x)^{n+1}}{\left(n+\beta_{1}\right)(n-1)}-\frac{2 \alpha_{1} x^{2}(1-x)^{n}}{n+\beta_{1}}\right] \\
& +\lambda_{1} \frac{2 n x-1-4 n x^{2}+(2 n+1) x^{n+1}+(1-x)^{n+1}+\alpha_{1}^{2}-4 \alpha_{1} x}{\left(n+\beta_{1}\right)^{2}(n-1)} \\
& +\lambda_{1} \frac{2 \alpha_{1} n-2 \alpha_{1}\left(\alpha_{1}+n\right)\left(x^{n+1}+(1-x)^{n}\right)+\alpha_{1}^{2} x\left(n^{2}+1\right)(1-x)^{n}}{\left(n+\beta_{1}\right)^{2}\left(n^{2}-1\right)} \\
B_{n, m}^{\lambda, \alpha, \beta}\left((t-y)^{2} ; x, y\right)= & \frac{m y(1-y)+\left(\beta_{2} y-\alpha_{2}\right)^{2}}{\left(m+\beta_{2}\right)^{2}} \\
& +\lambda_{2}\left[\frac{4 y^{2}-2 y-2 y^{m+2}-2\left(\alpha_{2}-1\right) y(1-y)^{m+1}}{\left(m+\beta_{2}\right)(m-1)}-\frac{2 \alpha_{2} y^{2}(1-y)^{n}}{m+\beta_{2}}\right] \\
& +\lambda_{2} \frac{2 m y-1-4 m y^{2}+(2 m+1) y^{m+1}+(1-y)^{m+1}+\alpha_{2}^{2}-4 \alpha_{2} y}{\left(m+\beta_{2}\right)^{2}(m-1)} \\
& +\lambda_{2} \frac{2 \alpha_{2} m-2 \alpha_{2}\left(\alpha_{2}+m\right)\left(y^{m+1}+(1-y)^{m}\right)+\alpha_{2}^{2} y\left(m^{2}+1\right)(1-y)^{m}}{\left(m+\beta_{2}\right)^{2}\left(m^{2}-1\right)} .
\end{aligned}
$$

Theorem 10. Let $f \in C(I)$. Then, the following inequality holds:

$$
\left|B_{n, m}^{\lambda, \alpha, \beta}(f ; x, y)-f(x, y)\right| \leq 2\left[\omega_{1}\left(f ; v_{n}^{1 / 2}(\alpha, \beta, \lambda ; x)\right)+\omega_{2}\left(f ; v_{n}^{1 / 2}(\alpha, \beta, \lambda ; y)\right)\right],
$$

where $v_{n}(\alpha, \beta, \lambda ; x)$ and $\nu_{m}(\alpha, \beta, \lambda ; y)$ are defined in Theorem 9. 
Proof. By using the definition of partial modulus of continuity and Cauchy-Schwartz inequality, we have

$$
\begin{aligned}
\left|B_{n, m}^{\lambda, \alpha, \beta}(f ; x, y)-f(x, y)\right| & B_{n, m}^{\lambda, \alpha, \beta}(|f(s, t)-f(x, y)| ; x, y) \\
\leq & B_{n, m}^{\lambda, \alpha, \beta}(|f(s, t)-f(x, t)| ; x, y)+B_{n, m}^{\lambda, \alpha, \beta}(|f(x, t)-f(x, y)| ; x, y) \\
\leq & B_{n, m}^{\lambda, \alpha, \beta}\left(\left|\omega_{1}(f ;|s-x|)\right| ; x, y\right)+B_{n, m}^{\lambda, \alpha, \beta}\left(\left|\omega_{2}(f ;|t-y|)\right| ; x, y\right) \\
\leq & \omega_{1}\left(f, v_{n}(\alpha, \beta, \lambda ; x)\right)\left[1+\frac{1}{v_{n}(\alpha, \beta, \lambda ; x)} B_{n, m}^{\lambda, \alpha, \beta}(|s-x| ; x, y)\right] \\
& +\omega_{2}\left(f, v_{m}(\alpha, \beta, \lambda ; y)\right)\left[1+\frac{1}{v_{m}(\alpha, \beta, \lambda ; y)} B_{n, m}^{\lambda, \alpha, \beta}(|t-y| ; x, y)\right] \\
\leq & \omega_{1}\left(f, v_{n}^{1 / 2}(\alpha, \beta, \lambda ; x)\right)\left[1+\frac{1}{v_{n}^{1 / 2}(\alpha, \beta, \lambda ; x)}\left(B_{n, m}^{\lambda, \alpha, \beta}\left((s-x)^{2} ; x, y\right)\right)^{1 / 2}\right] \\
& +\omega_{2}\left(f, v_{n}^{1 / 2}(\alpha, \beta, \lambda ; x)\right)\left[1+\frac{1}{v_{m}^{1 / 2}(\alpha, \beta, \lambda ; y)}\left(B_{n, m}^{\lambda, \alpha, \beta}\left((t-y)^{2} ; x, y\right)\right)^{1 / 2}\right] .
\end{aligned}
$$

Finally, by choosing $v_{n}(\alpha, \beta, \lambda ; x)$ and $v_{m}(\alpha, \beta, \lambda ; y)$ as defined in Theorem 9, we obtain desired result.

We recall that the Lipschitz class $\operatorname{Lip}_{M}\left(\widehat{\beta}_{1}, \widehat{\beta}_{2}\right)$ for the bivariate is given by

$$
|f(s, t)-f(x, y)| \leq M|s-x|^{\widehat{\beta}_{1}}|t-y|^{\widehat{\beta}_{2}}
$$

for $\widehat{\beta}_{1}, \widehat{\beta}_{2} \in(0,1]$ and $(s, t),(x, y) \in I_{a b}$.

Theorem 11. Let $f \in \operatorname{Lip}\left(\widehat{\beta}_{1}, \widehat{\beta}_{2}\right)$. Then, for all $(x, y) \in I_{a b}$, we have

$$
\left|B_{n, m}^{\lambda, \alpha, \beta}(f ; x, y)-f(x, y)\right| \leq M v_{n}^{\widehat{\beta}_{1} / 2}(\alpha, \beta, \lambda ; x) v_{m}^{\widehat{\beta}_{2} / 2}(\alpha, \beta, \lambda ; y),
$$

where $v_{n}(\alpha, \beta, \lambda ; x)$ and $v_{m}(\alpha, \beta, \lambda ; y)$ are defined in Theorem 9.

Proof. We have

$$
\begin{aligned}
&\left|B_{n, m}^{\lambda, \alpha, \beta}(f ; x, y)-f(x, y)\right| \leq B_{n, m}^{\lambda, \alpha, \beta}(|f(s, t)-f(x, y)| ; x, y) \\
& \leq M B_{n, m}^{\lambda, \alpha, \beta}\left(|s-x|^{\hat{\beta}_{1}}|t-y|^{\widehat{\beta}_{2}} ; x, y\right) \\
&=M B_{n, m}^{\lambda, \alpha, \beta}\left(|s-x|^{\widehat{\beta}_{1}} \mid ; x, y\right) B_{n, m}^{\lambda, \alpha, \beta}\left(|t-y|^{\widehat{\beta}_{2}} ; x, y\right)
\end{aligned}
$$

since $f \in \operatorname{Lip}_{M}\left(\widehat{\beta}_{1}, \widehat{\beta}_{2}\right)$. Then, by applying the Hölder's inequality for

$$
\widehat{p}_{1}=\frac{2}{\widehat{\beta}_{1}}, \widehat{q}_{1}=\frac{2}{2-\widehat{\beta}_{1}}
$$

and

$$
\widehat{p}_{2}=\frac{1}{\widehat{\beta}_{2}}, \widehat{q}_{2}=\frac{2}{2-\widehat{\beta}_{2}}
$$


we obtain

$$
\begin{aligned}
\left|B_{n, m}^{\lambda, \alpha, \beta}(f ; x, y)-f(x, y)\right| \leq & M\left\{B_{n, m}^{\lambda, \alpha, \beta}\left(|s-x|^{2} ; x, y\right)\right\}^{\widehat{\beta}_{1} / 2}\left\{B_{n, m}^{\lambda, \alpha, \beta}(1 ; x, y)\right\}^{\widehat{\beta}_{1} / 2} \\
& \times\left\{B_{n, m}^{\lambda, \alpha, \beta}\left(|t-y|^{2} ; x, y\right)\right\}^{\widehat{\beta}_{2} / 2}\left\{B_{n, m}^{\lambda, \alpha, \beta}(1 ; x, y)\right\}^{\widehat{\beta}_{2} / 2} \\
& =M v_{n}(\alpha, \beta, \lambda ; x)^{\widehat{\beta}_{1} / 2} v_{m}(\alpha, \beta, \lambda ; y)^{\widehat{\beta}_{2} / 2} .
\end{aligned}
$$

This completes the proof.

Theorem 12. For $f \in C^{1}(I)$, the following inequality holds:

$$
\left|B_{n, m}^{\lambda, \alpha, \beta}(f ; x, y)-f(x, y)\right| \leq\left\|f_{x}\right\|_{C(I)} v_{n}^{1 / 2}(\alpha, \beta, \lambda ; x)+\left\|f_{y}\right\|_{C(I)} v_{m}^{1 / 2}(\alpha, \beta, \lambda ; y),
$$

where $v_{n}(\alpha, \beta, \lambda ; x)$ and $v_{m}(\alpha, \beta, \lambda ; y)$ are defined in Theorem 9.

Proof. We have

$$
f(t)-f(s)=\int_{x}^{t} f_{u}(u, s) d u+\int_{y}^{s} f_{v}(x, v) d u
$$

for $(s, t) \in I$. Thus, by applying the operators defined in (24) to the above equality, we obtain

$$
\begin{aligned}
& \left|B_{n, m}^{\lambda, \alpha, \beta}(f ; x, y)-f(x, y)\right| \\
& \quad \leq B_{n, m}^{\lambda, \alpha, \beta}\left(\left|\int_{x}^{t} f_{u}(u, s) d u\right| ; x, y\right)+B_{n, m}^{\lambda, \alpha, \beta}\left(\left|\int_{y}^{s} f_{v}(x, v) d u\right| ; x, y\right) .
\end{aligned}
$$

By taking the following relations into our consideration

$$
\left|\int_{x}^{t} f_{u}(u, s) d u\right| \leq\left\|f_{x}\right\|_{C\left(I_{a b}\right)}|s-x|
$$

and

$$
\left|\int_{y}^{s} f_{v}(x, v) d u\right| \leq f_{y} \|_{C\left(I_{a b}\right)}|t-y|
$$

one obtains

$$
\begin{aligned}
& \left|B_{n, m}^{\lambda, \alpha, \beta}(f ; x, y)-f(x, y)\right| \\
& \quad \leq\left\|f_{x}\right\|_{C(I)} B_{n, m}^{\lambda, \alpha, \beta}(|s-x| ; x, y)+\left\|f_{y}\right\|_{C(I)} B_{n, m}^{\lambda, \alpha, \beta}(|t-y| ; x, y) .
\end{aligned}
$$

Using Cauchy-Schwarz inequality, we have

$$
\begin{aligned}
& \left|B_{n, m}^{\lambda, \alpha, \beta}(f ; x, y)-f(x, y)\right| \\
& \quad \leq\left\|f_{x}\right\|_{C(I)}\left\{B_{n, m}^{\lambda, \alpha, \beta}\left((s-x)^{2} ; x, y\right)\right\}^{1 / 2}\left\{B_{n, m}^{\lambda, \alpha, \beta}(1 ; x, y)\right\}^{1 / 2} \\
& \quad+\left\|f_{y}\right\|_{C(I)}\left\{B_{n, m}^{\lambda, \alpha, \beta}\left((t-y)^{2} ; x, y\right)\right\}^{1 / 2}\left\{B_{n, m}^{\lambda, \alpha, \beta}(1 ; x, y)\right\}^{1 / 2}
\end{aligned}
$$

Finally, we presents a Voronovskaja-type theorem for $B_{n, n}^{\lambda, \alpha, \beta}(f ; x, y)$. 
Theorem 13. Let $f \in C^{2}(I)$. Then

$$
\begin{aligned}
\lim _{n \rightarrow \infty} n\left[B_{n, n}^{\lambda, \alpha, \beta}(f ; x, y)-f(x, y)\right]= & \left(\alpha_{1}-\beta_{1} x\right) f_{x}+\left(\alpha_{2}-\beta_{2} y\right) f_{y} \\
& +\frac{x(1-x)}{2} f_{x x}+\frac{y(1-y)}{2} f_{y y} .
\end{aligned}
$$

Proof. Let $(x, y) \in I$ and write the Taylor's formula of $f(s, t)$ as

$$
\begin{aligned}
f(s, t)= & f(x, y)+f_{x}(s-x)+f_{y}(t-y) \\
& +\frac{1}{2}\left\{f_{x x}(s-x)^{2}+2 f_{x y}(s-x)(t-y)+f_{y y}(t-y)^{2}\right\} \\
& +\varepsilon(s, t)\left((s-x)^{2}+(t-y)^{2}\right)
\end{aligned}
$$

where $(s, t) \in I$ and $\varepsilon(s, t) \longrightarrow 0$ as $(s, t) \longrightarrow(x, y)$. If we apply sequence of operators $B_{n, n}^{\lambda, \alpha, \beta}(\cdot ; x, y)$ on (25) keeping in mind linearity of operator, we have

$$
\begin{aligned}
B_{n, n}^{\lambda, \alpha, \beta} & (f ; s, t)-f(x, y) \\
= & f_{x}(x, y) B_{n, n}^{\lambda, \alpha, \beta}((s-x) ; x, y)+f_{y}(x, y) B_{n, n}^{\lambda, \alpha, \beta}((t-y) ; x, y) \\
& +\frac{1}{2}\left\{f_{x x} B_{n, n}^{\lambda, \alpha, \beta}\left((s-x)^{2} ; x, y\right)+2 f_{x y} B_{n, n}^{\lambda, \alpha, \beta}((s-x)(t-y) ; x, y)\right. \\
& \left.+f_{y y} B_{n, n}^{\lambda, \alpha, \beta}\left((t-y)^{2} ; x, y\right)\right\}+B_{n, n}^{\lambda, \alpha, \beta}\left(\varepsilon(s, t)\left((s-x)^{2}+(t-y)^{2}\right) ; x, y\right) .
\end{aligned}
$$

Applying limit to both sides of the last equality as $n \rightarrow \infty$, we have

$$
\begin{aligned}
\lim _{n \rightarrow \infty} n\left(B_{n, n}^{\lambda, \alpha, \beta}(f ; s, t)-f(x, y)\right) \\
=\lim _{n \rightarrow \infty} n\left\{f_{x}(x, y) B_{n, n}^{\lambda, \alpha, \beta}((s-x) ; x, y)+f_{y}(x, y) B_{n, n}^{\lambda, \alpha, \beta}((t-y) ; x, y)\right\} \\
\quad+\lim _{n \rightarrow \infty} \frac{n}{2}\left\{f_{x x} B_{n, n}^{\lambda, \alpha, \beta}\left((s-x)^{2} ; x, y\right)\right. \\
\left.\quad+2 f_{x y} B_{n, n}^{\lambda, \alpha, \beta}((s-x)(t-y) ; x, y)+f_{y y} B_{n, n}^{\lambda, \alpha, \beta}\left((t-y)^{2} ; x, y\right)\right\} \\
\quad+\lim _{n \rightarrow \infty} n B_{n, n}^{\lambda, \alpha, \beta}\left(\varepsilon(s, t)\left((s-x)^{2}+(t-y)^{2}\right) ; x, y\right) .
\end{aligned}
$$

Using Hölder inequality for the last term of above equality, we have

$$
\begin{aligned}
& B_{n, n}^{\lambda, \alpha, \beta}\left(\varepsilon(s, t)\left((s-x)^{2}+(t-y)^{2}\right) ; x, y\right) \\
& \leq \sqrt{2} \sqrt{B_{n, n}^{\lambda, \alpha, \beta}\left(\varepsilon^{2}(s, t) ; x, y\right)} \\
& \quad \times \sqrt{B_{n, n}^{\lambda, \alpha, \beta}\left(\varepsilon(s, t)\left((s-x)^{4}+(t-y)^{4}\right) ; x, y\right)} .
\end{aligned}
$$

Since

$$
\lim _{n \rightarrow \infty} B_{n, n}^{\lambda, \alpha, \beta}\left(\varepsilon^{2}(s, t) ; x, y\right)=\varepsilon^{2}(x, y)=0
$$

we have

$$
\lim _{n \rightarrow \infty} n B_{n, n}^{\lambda, \alpha, \beta}\left(\varepsilon(s, t)\left((s-x)^{4}+(t-y)^{4}\right) ; x, y\right)=0 .
$$


Consequently, we obtain

$$
\begin{gathered}
\lim _{n \rightarrow \infty} n B_{n, n}^{\lambda, \alpha, \beta}((s-x) ; x, y)=\alpha_{1}-\beta_{1} x \\
\lim _{n \rightarrow \infty} n B_{n, n}^{\lambda, \alpha, \beta}((t-y) ; x, y)=\alpha_{2}-\beta_{2} y, \\
\lim _{n \rightarrow \infty} n B_{n, n}^{\lambda, \alpha, \beta}\left((s-x)^{2} ; x, y\right)=x(1-x), \\
\lim _{n \rightarrow \infty} n B_{n, n}^{\lambda, \alpha, \beta}\left((t-y)^{2} ; x, y\right)=y(1-y) .
\end{gathered}
$$

Combining (26)-(30), we deduce the desired result.

Author Contributions: All authors contributed equally in this work.

Funding: This research received no external funding

Conflicts of Interest: The authors declare no conflict of interest.

\section{References}

1. Bernstein, S.N. Démonstration du théorème de Weierstrass fondée sur le calcul des probabilités. Comm. Soc. Math. Kharkow 1913, 13, 1-2.

2. Stancu, D.D. Asupra unei generalizari a polinoamelor lui Bernstein. Studia Univ. Babes-Bolyai Ser. Math.-Phys. $1969,14,31-45$.

3. Acar, T.; Mohiuddine, S.A.; Mursaleen, M. Approximation by $(p, q)$-Baskakov-Durrmeyer-Stancu operators. Complex Anal. Oper. Theory 2018, 12, 1453-1468. [CrossRef]

4. Baxhaku, B.; Agrawal, P.N. Degree of approximation for bivariate extension of Chlodowsky-type $q$-Bernstein-Stancu-Kantorovich operators. Appl. Math. Comput. 2017, 306, 56-72. [CrossRef]

5. Chauhan, R.; Ispir, N.; Agrawal, P.N. A new kind of Bernstein-Schurer-Stancu-Kantorovich-type operators based on $q$-integers. J. Inequal. Appl. 2017, 2017, 50. [CrossRef] [PubMed]

6. Mursaleen, M.; Ansari, K.J.; Khan, A. Some approximation results by $(p, q)$-analogue of Bernstein-Stancu operators. Appl. Math. Comput. 2015, 264, 392-402. [CrossRef]

7. Cai, Q.-B.; Lian, B.-Y.; Zhou, G. Approximation properties of $\lambda$-Bernstein operators, J. Inequal. Appl. 2018, 2018, 61. [CrossRef] [PubMed]

8. Ye, Z.; Long, X.; Zeng, X.-M. Adjustment algorithms for Bézier curve and surface. In Proceedings of the International Conference on Computer Science and Education, Hefei, China, 24-27 August 2010; pp. 1712-1716.

9. Cai, Q.-B. The Bézier variant of Kantorovich type $\lambda$-Bernstein operators. J. Inequal. Appl. 2018, $2018,90$. [CrossRef] [PubMed]

10. Acu, A.M.; Manav, N.; Sofonea, D.F. Approximation properties of $\lambda$-Kantorovich operators. J. Inequal. Appl. 2018, 2018, 202. [CrossRef]

11. Özger, F. Some general statistical approximation results for $\lambda$-Bernstein operators. arXiv 2018, arXiv:1901.01099.

12. Acar, T.; Aral, A. On pointwise convergence of $q$-Bernstein operators and their $q$-derivatives. Numer. Funct. Anal. Optim. 2015, 36, 287-304. [CrossRef]

13. Acar, T.; Aral, A.; Mohiuddine, S.A. On Kantorovich modification of $(p, q)$-Bernstein operators. Iran. J. Sci. Technol. Trans. Sci. 2018, 42, 1459-1464. [CrossRef]

14. Acar, T.; Aral, A.; Mohiuddine, S.A. Approximation by bivariate $(p, q)$-Bernstein-Kantorovich operators. Iran. J. Sci. Technol. Trans. Sci. 2018, 42, 655-662. [CrossRef]

15. Acar, T.; Aral, A.; Mohiuddine, S.A. On Kantorovich modification of $(p, q)$-Baskakov operators. J. Inequal. Appl. 2016, 2016, 98. [CrossRef]

16. Acu, A.M.; Muraru, C. Approximation properties of bivariate extension of $q$-Bernstein-Schurer-Kantorovich operators. Results Math. 2015, 67, 265-279. [CrossRef]

17. Mishra, V.N.; Patel, P. On generalized integral Bernstein operators based on $q$-integers. Appl. Math. Comput. 2014, 242, 931-944. [CrossRef] 
18. Mohiuddine, S.A.; Acar, T.; Alotaibi, A. Construction of a new family of Bernstein-Kantorovich operators. Math. Methods Appl. Sci. 2017, 40, 7749-7759. [CrossRef]

19. Mohiuddine, S.A.; Acar, T.; Alotaibi, A. Durrmeyer type $(p, q)$-Baskakov operators preserving linear functions. J. Math. Inequal. 2018, 12, 961-973. [CrossRef]

20. Mohiuddine, S.A.; Acar, T.; Alghamdi, M.A. Genuine modified Bernstein-Durrmeyer operators. J. Inequal. Appl. 2018, 2018, 104. [CrossRef] [PubMed]

21. Mursaleen, M.; Ansari, K.J.; Khan, A. On ( $p, q)$-analogue of Bernstein operators. Appl. Math. Comput. 2018, 266 , 874-882; Erratum in Appl. Math. Comput. 2016, 278, 70-71. [CrossRef]

22. Braha, N.L.; Srivastava, H.M.; Mohiuddine, S.A. A Korovkin's type approximation theorem for periodic functions via the statistical summability of the generalized de la Vallée Poussin mean. Appl. Math. Comput. 2014, 228, 62-169. [CrossRef]

23. Srivastava, H.M.; Zeng, X.-M. Approximation by means of the Szász-Bézier integral operators. Int. J. Pure Appl. Math. 2004, 14, 283-294.

24. Ditzian, Z.; Totik, V. Moduli of Smoothness; Springer: New York, NY, USA, 1987.

25. DeVore, R.A.; Lorentz, G.G. Constructive Approximation; Springer: Berlin, Germany, 1993.

26. Peetre, J. A Theory of Interpolation of Normed Spaces; Notas Mat.: Rio de Janeiro, Brazil, 1963.

27. Ozarslan, M.A.; Aktuğlu, H. Local approximation for certain King type operators. Filomat 2013, 27, $173-181$. [CrossRef]

28. Büyükyazıcı, İ; İbikli, E. The properties of generalized Bernstein polynomials of two variables. Appl. Math. Comput. 2004, 156, 367-380. [CrossRef]

29. Martinez, F.L. Some properties of two-demansional Bernstein polynomials. J. Approx. Theory 1989, 59, 300-306. [CrossRef]

30. Volkov, V.J. On the convergence of linear positive operators in the space of continuous functions of two variables. Doklakad Nauk SSSR 1957, 115, 17-19.

(C) 2019 by the authors. Licensee MDPI, Basel, Switzerland. This article is an open access article distributed under the terms and conditions of the Creative Commons Attribution (CC BY) license (http:/ / creativecommons.org/licenses/by/4.0/). 